\title{
Degrees of competency: the relationship between educational qualifications and adult skills across countries
}

\author{
Natascha Massing ${ }^{*}$ (10) and Silke L. Schneider
}

\author{
*Correspondence: \\ natascha.massing@gesis.org \\ GESIS - Leibniz Institute \\ for the Social Sciences, B2, 1, \\ 68159 Mannheim, Germany
}

\begin{abstract}
Background: Educational qualifications and literacy skills are highly related. This is not surprising as it is one aim of educational systems to equip individuals with competencies necessary to take part in society. Because of this relationship educational qualifications are often used as a proxy for "human capital". However, from a theoretical perspective, there are many reasons why this relationship is not perfect, and to some degree this is due to third variables. Thus, we want to explore the net relationship between educational attainment (harmonized according to the International Standard Classification of Education, ISCED) and literacy skills, and how much skills vary within education levels across countries.
\end{abstract}

Methods: We use data from 21 countries from the Programme for the International Assessment of Adult Competencies 2012. This paper compares the literacy skills of adults who achieved different levels of educational attainment across countries. Given the high degree of educational differentiation in most countries, we do this using a more differentiated educational attainment variable than what is commonly used. In our analyses we firstly adjust for factors that are likely to affect access to education and the acquisition of educational qualifications and literacy skills, such as parental education and language and migration background. In a second step, we also take into account factors affecting skill development after initial formal education, such as occupation and skill use at home.

Results: We firstly find a high degree of heterogeneity of skills across countries for equivalent education categories. Secondly, we find skill similarities for equivalent education categories classified at different broad education levels, sometimes even breaking the hierarchical order of 'higher education entails higher competencies'.

Conclusion: We conclude that ISCED levels cannot be taken as a cross-nationally comparable proxy for human capital in terms of literacy skills, and that education has to be harmonized in a substantively more meaningful way in future adult literacy surveys.

\section{Background}

Educational attainment and how it relates to social and migration background as well as labor market outcomes has been studied extensively using comparative data and methods (see for example Breen and Jonsson 2005; Heath and Brinbaum 2014; Shavit and Blossfeld 1993; Shavit and Müller 1998). Due to limited data on nationally representative 
adult samples, there is much less cross-national research on these relationships taking basic competencies or skills into account. Research using large-scale assessment data of the adult population across countries has shown that education is a key determinant of adult basic competencies (Maehler et al. 2013; OECD 2013a; OECD and Statistics Canada 2000b, 2005). This is not surprising as one aim of formal education is to develop basic competencies in order to prepare students for life and specifically the job market.

However, OECD (2013a) and other authors (Desjardins 2003; Park and Kyei 2011; Reder 2009) also show that the relationship between educational qualifications and skills is imperfect, meaning that formal education does not entirely explain skill differences amongst adults, and other factors must also play an important role. Thus we agree that "better understanding the link between formal qualifications and actual skills is important because qualifications are more readily observable than skills and therefore often serve as an important proxy for the latter" (Heisig and Solga 2015, p. 203). The use of education as a proxy for skills is especially common when arguing from the point of view of human capital theory (Becker 1964; Steedman and Murray 2001) and other functionalist approaches to education. ${ }^{1}$ However, there is also longstanding opposition to the functionalist view of education, claiming that education also or even mostly reflects the distribution of power in society (e.g., Collins 1971). In fact, large-scale assessments are largely motivated by the desire to more directly measure adult competencies than using educational attainment as a proxy.

In addition, policy makers may want to know whether individuals who completed a given level of education show the same level of competencies in their country as in other countries, or whether they 'lag behind'. They are also keen to find out whether migrants from other countries possess similar competency levels as natives at comparable levels of educational attainment. Comparable or equivalent education levels are typically defined by the International Standard Classification of Education [ISCED; (UNESCO Institute for Statistics 2006)] used in many cross-national surveys. We however do not yet know very much about how and why competencies differ across countries for the same, supposedly comparable, education levels (but see Heisig and Solga 2015; Park and Kyei 2011).

Prior research looking at the relationship between formal education and competencies (for example, Desjardins 2003; OECD 2013a; OECD and Statistics Canada 2000a, 2005) found that educational attainment shows the strongest relationship with competencies of all background variables ${ }^{2}$ examined, when adjusting for other socio-demographic factors. At the same time, there are substantial differences across countries in average proficiency levels at the same broad level of educational attainment (Maehler et al. 2013; OECD 2013a; OECD and Statistics Canada 2005). Part of the OECD (2013a, chapter 5) report also looks more closely into different factors that may affect adult literacy skills beyond demographics, which is an important analysis step. For example, if the higher educated show comparatively low literacy skills in one country, this may be due to many

\footnotetext{
${ }^{1}$ A notable exception is some literature on educational and skill mismatches in the labor market, which critically examines the relationship between educational certificates and actual skills (Allen and Van der Velden 2001; Green and McIntosh 2007).

${ }^{2}$ It is interesting to note that the OECD treats educational attainment as a mere 'socio-demographic' variable (just as socio-economic background, another important factor in skill development).
} 
of them working in occupations not nurturing competencies, i.e. the structure of the labor market, rather than ineffective formal education or low selectivity of educational transitions by skills. This kind of confounding is also referred to as compositional effects in the literature (Raudenbush and Kim 2002). Therefore, when trying to compare the relationship between formal education and competencies across countries, it is important to adjust for these factors: otherwise, cross-country differences could come about through mere differences in the composition of countries according to e.g. parental education or respondents' occupation since some countries have more expanded educational systems than others, and they differ in their industrial and thus occupational structures.

Furthermore, the OECD, as most reports in official statistics, mostly uses three very broad education levels derived from the International Standard Classification of Education (ISCED) 1997, by only distinguishing between (1) less than upper secondary, (2) upper secondary and post-secondary non-tertiary and (3) tertiary levels of education. Academic studies (for example Heisig and Solga 2015; Park and Kyei 2011) often follow this approach. Sometimes the investigation is even limited to the contrast between (1) less than upper secondary and (3) tertiary education. However, we know from previous research that such highly aggregated education measures may not be valid measures of attainment within countries (Müller and Klein 2008), and may, as a consequence, be cross-nationally not actually comparable (Schneider 2010). For example, if upper secondary graduates in one country are largely graduates of vocationally oriented programs, and in another country of academically oriented programs, lumping them together in one education category for analysis does not help our understanding of differences and similarities in outcomes of basic skill acquisition and educational selection across these two countries. Therefore, before jumping to conclusions about differences in the quality and academic selectivity of education across countries, it is important to describe 'net' skill differences by disaggregated, cross-nationally more comparable educational attainment categories than has so far been done.

With our analyses we want to broaden the scope of the analyses presented by the OECD in 2013 based on data from the Programme of International Assessment of Adult Competencies (PIAAC) (OECD 2013a, chapter 3). In doing so, we build on related work by Desjardins (2003), Park and Kyei (2011), Maehler et al. (2013) and Heisig and Solga (2015). This paper has two aims: First of all, we describe how detailed educational attainment relates to literacy skills across different countries. Secondly, we explore how far cross-country differences in skills by detailed educational attainment remain or change when adjusting for a wide range of micro-level variables likely to influence educational attainment and/or adult competencies. We will thereby be able to approximate 'net' cross-country differences and similarities in competencies by educational attainment.

With the results we hope to be able to answer the following research questions: Firstly, how closely are adult basic competencies related to educational attainment across countries? Are the competencies of individuals who have achieved 'comparable' levels of education also comparable, adjusting for factors related to the acquisition of formal education and basic competencies? Do we find the same differences in skills across countries already identified in the OECD reports when looking at detailed rather than highly aggregated education levels and controlling for a wide range of individual level variables? 
If we find substantial differences, these potentially point to (a) differences in the quality (effectiveness of skill acquisition) and skill selectivity of education between countries, (b) substantive lack of comparability of harmonized education categories regarding competencies as one outcome of formal education or (c) omission of important further confounding factors influencing educational attainment and competencies.

We first describe the theoretical relationship between detailed educational attainment categories and competencies, reviewing the literature and evidence in the field. Then, we turn to the data, measures and methods we use. In our results we show how adult competency scores of groups with 'comparable' educational qualifications vary between countries. We include several variables in order to disentangle which other factors could influence this relationship. We summarize our findings and discuss them in relation to potential improvements when measuring educational qualification in surveys, as well as opportunities for further research.

\section{The relationship between educational qualifications and competencies}

Competencies "...refer to the ability or capacity of an agent to act appropriately in a given situation” (OECD 2013b), especially to someone's proficiency in performing certain tasks. Competencies “... represent skills essential for accessing, understanding, analyzing and using text-based information and, in the case of some mathematical information, information in the form of representations (e.g. pictures, graphs)" (OECD 2013b). We use the terms 'competency' and 'skill' interchangeably in this study.

Although the specific competencies measured in large-scale assessments can be expected to be closely related to general cognitive ability or fluid intelligence, and some authors treat them as almost exchangeable (Kerckhoff et al. 2001; Marks 2014) these two concepts are theoretically and empirically distinct. Most importantly, competencies are conceptualized as domain specific skills, focusing e.g. on literacy or numeracy, whereas fluid intelligence refers to generalized cognitive functioning (Baumert et al. 2009).

While competencies are understood as a continuum and typically unobserved latent characteristics, educational qualifications, going along with receiving a formal diploma, certificate or an academic title, reflect manifest thresholds or steps in the educational career. Having achieved an educational qualification usually confers some explicit opportunity or entitlement to the holder of the qualification, e.g. the opportunity to enroll in a university or (further qualify to) practice a specific occupation. Educational qualifications correspond to 'institutionalized' cultural capital (Bourdieu 1986). They allow the individual to objectify their embodied cultural capital, which includes competencies, and "makes the difference between the capital of the autodidact, which may be called into question at any time [...] and the cultural capital academically sanctioned by legally guaranteed qualifications, formally independent of the person of their bearer" (Bourdieu 1986). Educational qualifications facilitate the conversion of cultural capital to economic capital. Qualifications also serve as signaling devices (Arrow 1973; Spence 1973) that employers, university admissions or other selecting agents can actually see when an applicant sends in copies of the diplomas and degrees she holds, whereas her actual competencies remain unobserved.

Basic competencies are "[...] the results of cumulative processes of knowledge acquisition that are moderated to some extent by reasoning ability" (Baumert et al. 2009, p. 
174). Many of these processes are facilitated by formal education. Therefore, the more opportunities for knowledge acquisition are provided to and used by an individual, the higher the level of formal education and basic competencies achieved. This point of view thus leads to the expectation that educational attainment and basic competencies are closely related. Indeed, using data from the International Adult Literacy Study (IALS), the Adult Literacy and Life Skills Survey (ALL) and PIAAC, OECD and Statistics Canada (2000b, 2005), Boudard (2001), Desjardins (2003) and OECD (2013a), among others, show that across countries, education has the strongest relationship with competencies of all background variables examined, confirming results from the US National Adult Literacy Survey (Kerckhoff et al. 2001; Kirsch et al. 1993).

However, as the imperfect correlation between educational attainment and competencies suggests, knowledge acquisition and competency formation are not limited to formal education (Desjardins 2003): competency development is an experience that is both "lifewide" (occurring in the home, at school, work and in the community) and "lifelong" (starting during fetal development and continuing into old age). Practice engagement theory (Reder 1994) posits that literacy is generally learned through engagement in literacy practices, which extend far beyond formal education. Also, some of the correlation may be spurious, i.e. due to common causes. In order to assess the net relationship between educational attainment and skills, we first need to theoretically reflect on factors affecting both success in education and skills (in the sense of common causes), as well as factors potentially depending on education further impacting skills (in the sense of mediators). Only after adjusting for these factors and thus controlling compositional differences across countries, we can try to better understand competency differences across countries within supposedly comparable education levels.

To a large extent, especially in the early years of life, competency formation, especially relating to language, takes place through informal learning or primary socialization in the family context. The family is also important in nourishing curiosity and motivation to learn in children. These early skills and attitudes to learning facilitate further competency gains and transitions in formal education. Therefore, the gross or total relationship between educational attainment and competencies will partly be due to opportunities for informal learning as well as attitudes to learning bred in the home, which differ across families. When estimating the net relationship between formal education and competencies, it is thus important to adjust for characteristics of the family of origin that likely influence their offspring's education and skills.

The literature discusses a diverse range of family characteristics when dealing with educational outcomes, which can mostly be attributed to three dimensions, namely genetic, cultural and economic resources. Firstly, cognitive abilities or general intelligence have been shown to correlate strongly between parents and their children (by 0.4-0.7, see the review by Marks 2014, chapter 4), and, using twin and adoption studies, to have a substantial degree of heritability, with monozygotic twins reared apart showing a correlation of cognitive abilities of around 0.7 (Marks 2014). This may be due to either genetic or pre-natal/pre-separation environmental commonalities though. At the same time, cognitive abilities positively influence competency formation and success in formal education (Marks 2014, chapter 5). However, only few studies have both measures of general cognitive abilities and later specific competencies, none of them cross-national. 
Therefore, we need to be aware that some of the effects of family characteristics that are described in the following will have some (maybe substantial) genetic component, and that some genetic effects remain unobserved.

Secondly, in terms of culture, parents' own educational attainment is regarded as the most important asset boosting offspring's educational opportunities (Erikson and Jonsson 1996; Shavit and Blossfeld 1993): more educated parents provide a more stimulating home environment to their children than less educated parents, for example by reading more to their children and using more complex language. They also have their own experience navigating the educational system and can thus better support their children in educational decision making, leading to higher levels of attainment. The influence of parents' education on adult literacy net of formal education was already found for the US by Kirsch et al. (1993), cross-nationally by OECD and Statistics Canada (2000a, 2005) and Desjardins (2003), and confirmed with PIAAC data by OECD (2013a). Bynner and Parsons (2009) find in their research using British cohort study data that family background has an effect on proficiency, which is mediated through earlier skill acquisition.

Closely related with parental education is the availability of books in the home when growing up, commonly used as an indicator of family's cultural capital in large-scale assessments. This factor mediates some of the effect of parental education, but also has an effect on top of that: families with low levels of formal education that nevertheless possess more books provide a more cognitively stimulating environment, especially more opportunities for engagement in literacy practice, for their children than families with no or fewer books (Evans et al. 2010). Children's reading practice has been shown to strongly support their reading competencies (Anderson et al. 1988). OECD (2013a) however does not control for the number of books in the home.

Another cultural family characteristic is migration background, which has often been shown to affect educational outcomes (Heath and Brinbaum 2014; Heath et al. 2008; Marks 2005; OECD 2012). First of all, respondents who were educated abroad may have had very different educational experiences, including different quality of basic education, in their country of origin. Secondly, first generation migrants and their children will lack knowledge of and first-hand experience with a country's educational system and thus may not navigate it in an optimal way. Thirdly, it can be assumed that respondents who were born in the survey country and are familiar with the survey language can more fully benefit from the learning opportunities provided to them in formal education than those born abroad or having a different mother tongue, positively contributing to both educational attainment and skills. Finally, the assessment is also language based so that respondents completing it in their native (and thus likely most proficient) language are expected to show higher competencies in literacy measured in this language. This has also been shown empirically before (Boudard 2001; Desjardins 2004; Elley 1992; Kirsch et al. 1993; OECD 2012; OECD and Statistics Canada 2000a, 2005). Using multivariate models and PIAAC data, Heisig and Solga (2015) as well as OECD (2013a) find substantial effects of migration background on numeracy and literacy skills, after adjusting for parental education, educational attainment and occupation.

Thirdly, in terms of economic circumstances, the most often-discussed family characteristic is social class or status (Breen et al. 2010; Erikson and Jonsson 1996; Shavit and Blossfeld 1993). It reflects the occupational position, economic security and material 
circumstances of the family, for example nutrition, housing and access to healthcare. Economically secured parents have more capacity to support their children's learning than those struggling to make ends meet. Some educational resources, such as a quiet place to study, books or out-of-school tutoring also have direct costs. Further family characteristics reflecting economic circumstances during childhood are parental income and wealth. Since these cannot be reliably measured in a survey interviewing the children's generation only, they are rarely used in empirical studies. Bynner and Parsons (2009) provide a vivid insight into social and material conditions of literacy skill development for Britain. Cross-national large-scale assessments have however shown considerably less interest in material than cultural conditions of competency development, using parental education as a proxy measure for 'socio-economic status' (following NALS, see Kirsch et al. 1993) instead of differentiating cultural and economic aspects, leading to a gap in comparative research on this issue.

Moving on to secondary socialization in the formal education system, higher early literacy skills facilitate learning and thus performance in education, the successful completion of an educational level, and making the transition to the next higher level of education. Earlier literacy skills are thus predictive of later literacy skills (Bynner and Parsons 2009). Therefore, the relationship between education and literacy is reciprocal: more literate individuals stay in education for longer and achieve a higher level of attainment, and staying in education for longer and reaching higher levels of attainment produce higher literacy (Kirsch et al. 1993). This reciprocity however cannot be disentangled with cross-sectional data lacking information on skills at earlier points in life (OECD and Statistics Canada 2005) so that to date, no cross-national evidence is available on this. What however can be disentangled with available data are differences in skills (whether coming about by differential skill selectivity or opportunities to learn and practice literacy) within broad education levels, namely those between attainment of vocational and non-vocational educational qualifications. Given that vocational programs focus on learning of vocational rather than basic skills such as literacy, and students with low literacy more likely select (or are selected into) vocational over generally or academically oriented courses, we expect average literacy skills related to vocational qualifications to be lower than those related to general qualifications (Heisig and Solga 2015). This was shown to be the case for most PIAAC countries allowing this analysis by Maehler et al. (2013).

Finally, competency acquisition does not end with the end of formal education but continues through the life course especially through work (and life) experience, opportunities for skill use, as well as deliberate efforts of life-long learning. Previous research has shown that literacy levels of individuals indeed change after the completion of educational qualifications (Reder 2009), and more so for respondents with non-manual jobs because they have been able to further develop their skills throughout their working lives (Steedman and McIntosh 2001). Opportunities for literacy skill use as well as adult training strongly differ across occupations or types of jobs, even after controlling for educational attainment (Desjardins et al. 2006; OECD and Statistics Canada 2005). Individuals in different occupational groups, even if measured inconsistently across studies, therefore show diverging literacy skills, on top of education, parental education, and language (Desjardins 2003; OECD 2013a; OECD and Statistics Canada 2000a). Also, not 
only the work context offers opportunities for skill use, and reading for leisure or other forms of literacy practice outside of work have also been shown to contribute to skill maintenance and enhancement after formal education both for the US (Smith 1996) as well as cross-nationally (Desjardins 2003; OECD 2013a, chapter 5; OECD and Statistics Canada 2000a). With respect to adult training, using IALS data, Park and Kyei (2011) find that training participation is related to literacy gaps. However, they only measured training participation at the country level. Desjardins (2003), OECD (2013a) and OECD and Statistics Canada (2000a, 2005) find that adult training has an-albeit weak-effect, on top of formal education and other variables, since training participation is strongly related to formal education.

Turning to cross-country differences in the relationship between educational attainment and competencies as well as skill gaps between education levels, OECD and Statistics Canada (2000a, 2005) and OECD (2013a) find that firstly, higher educational attainment goes along with higher competencies, but secondly, that there are marked differences in average competency scores across countries for equivalent levels of education, as well as in the competency gaps between education levels. Differences in skills across countries are more pronounced for the low than the highly educated. Using multivariate models, Park and Kyei (2011) find that in all countries, individuals with higher qualifications have higher literacy skills, as measured in IALS. They also find differences in average literacy across countries for comparable education levels. Again, the differences are more pronounced at the lower than the higher education level. The OECD report (2013a) provides more detailed multivariate results in chapter 5 , which does not substantially change the result: formal education is still considerably related to adult competencies, and literacy skills still vary substantially across countries for equivalent levels of formal education (even if less so than in the unadjusted model). In line with this, Heisig and Solga (2015), using PIAAC data, find that respondents with completed upper secondary education generally acquire higher numeracy scores than respondents with lower educational qualifications, and that some of the variation between countries is related to compositional effects. However, the latter only adjust for age, sex, and migration/language status.

Available studies to date only look at skill gaps between the high and low educated (OECD 2013a, chapter 3), between the medium and the low educated (Heisig and Solga 2015) or between low, medium and high educated (Park and Kyei 2011), ignoring the heterogeneity of educational programs and qualifications within these broad levels. When unpacking broad education levels into more detailed educational attainment categories, as suggested by Schneider (2010), important cross-country differences but also previously hidden similarities may emerge. Different distributions of education within broad education categories, such as vocationally educated individuals dominating the medium educated in one country and generally educated individuals dominating the medium educated in another country, may partly explain why the same broad education levels show different average literacy skills across countries, or why some countries show surprisingly small skill gaps between educational groups (look e.g. at Switzerland and Germany in Park and Kyei 2011) or low overall associations between attainment and competencies (for example, see the weak associations for Sweden, the Czech Republic and Germany in OECD and Statistics Canada 2000a, all countries with substantial 
differentiation of education within broad levels). In some analyses, the OECD (2013a, pp. 200-205) report actually looks at some of those more detailed differences by looking at differences between respondents with vocational vs. general upper secondary education, and type of education at the tertiary level, but only for age groups that are potentially still in education. Maehler et al. (2013) report competency by detailed education categories for Germany, finding that on average, individuals with vocational tertiary education achieve lower competency levels than individuals with general upper secondary education, contradicting the general finding of higher competencies at higher education levels found using broad education categories. In this paper we argue that such detailed analyses should be the rule rather than the exception because detailed educational attainment categories are more substantively comparable across countries and easier to interpret than broad education levels.

Summing up, we expect that parents' education as well as migration background has an influence on the way competencies are developed. It is likely that part of this effect is mediated through educational qualifications. Furthermore, we expect individuals working in occupations requiring higher literacy skills, individuals who regularly read at home, and those who participate in adult training to better sustain or even further develop their competencies than individuals who do not work, work in manual occupations, do not read at home, and do not participate in adult training. Because all these factors are likely to be influenced by educational attainment, we furthermore expect the skills gaps by education to be further reduced when taking post-educational experiences into account. Regarding the cross-country comparison of literacy skills by detailed educational attainment, we expect substantial differences in competencies for equivalent education categories even when adjusting for the above micro-level factors: equivalent educational programs (as defined by ISCED) in different countries differ in both skill selectivity upon entrance, as well as effectiveness of skill development.

\section{Data and methods}

For this paper, we use data from the Programme for the International Assessment of Adult Competencies (PIAAC) 2012 (OECD 2013a, 2013c). PIAAC is an international study which assessed central basic skills which are considered essential for successful participation in today's society: literacy, numeracy and problem-solving in technologyrich environments. In this paper, we concentrate on literacy, defined as "[...] understanding, evaluating, using and engaging with written texts to participate in society, to achieve one's goals, and to develop one's knowledge and potential" (PIAAC Literacy Expert Group 2009). In the first round of PIAAC, 24 countries took part. The focus of the study was on the working-age population between the ages of 16 and 65 . In each country, respondents for PIAAC were chosen using probability based methods, thus aiming at a representative sample of the population. In addition to this, PIAAC data benefitted from a high degree of input harmonization and other high quality control standards.

We restrict the sample to respondents aged 25 and older because in many countries, respondents are still in their initial phase of education and not yet highly involved with the labor market when they are younger than 25 years (see also Desjardins 2003). In contrast to Park and Kyei (2011), we do not restrict our sample to young adults in an attempt to eliminate the effects of post-educational learning. Instead, we take advantage of the 
measures of continuing training, occupational group and private reading habits available in PIAAC (see below) and analyze the whole PIAAC sample. Finally, in comparison Park and Kyei (2011) we do not restrict the sample to respondents born in the country. Instead we exclude respondents who have completed their highest education abroad as the aim is to measure the relationship between education and measured competencies within various educational systems. ${ }^{3}$ For our analyses we include 21 countries. We excluded data from the Russian Federation as the data is not representative for the whole resident population of the Russian Federation (OECD 2013a). We also exclude Australia, since this data is not publicly available, and Cyprus, because results showed unusual patterns, shedding substantial doubt on comparability with the other countries, as well as the high degree of literacy related non-response (LRNR) in Cyprus, meaning the nonparticipation because of language difficulties, or learning or mental disabilities (see Heisig and Solga 2015).

\section{Measures used}

Competencies (or skills) are measured using psychometric tests based on IRT scaling procedures (OECD 2013c). As mentioned above, in PIAAC, three different competency domains were measured: literacy, numeracy and problem-solving in technology-rich environments. The results of the scaling produced one scale for each of the domains assessed. Each of these scales ranges from 0 to 500. Tasks at the lower end of the scale are easier than those at the higher end. In order to make interpretation of the scales easier, each scale was divided into proficiency levels with intervals of 50 points (Levels 1-5 for literacy and numeracy and levels $1-3$ for problem-solving in technology-rich environment, OECD 2013b). For the purpose of this study, we have opted for literacy (rather than numeracy) because it is the more generally needed competency. As such, the acquisition of further skills such as numeracy to some degree presupposes literacy, and most of the numeracy test items in PIAAC were text based, too. Both scales are thus highly correlated.

Educational qualifications are measured by directly asking respondents for the highest qualification they have obtained or level of education completed, using a country-specific show card representing the relevant responses in any given country. The resulting categories are harmonized into a common scheme, which is based on the International Standard Classification of Education (ISCED) 1997 (UNESCO Institute for Statistics 2006). ISCED 1997 main levels are known for their heterogeneity and thus risk of lacking validity for cross-national comparisons (Schneider 2009, 2010). Therefore in this study, we do not employ ISCED main levels but code the detailed ISCED variable available in the PIAAC data in such a way as to render the resulting categories less heterogeneous.

Not all countries provided the ISCED information in the same level of detail. With the PIAAC data it is not possible to distinguish program destinations "A", "B" or "C" at ISCED levels 3 and 4 . Instead, we distinguish whether the qualification is vocationally

\footnotetext{
${ }^{3}$ In order to compute this information, the age when completing the highest degree as well as the age of immigration was needed. However, Germany, Canada, Estonia, and the United States did not provide this information as continuous variable. For the three latter countries, the information had to be derived from the categorized age and can therefore only be treated as proxy. For Germany, the continuous variable from the German Scientific Use File (Rammstedt et al. 2015) was used.
} 
oriented or not (including all qualifications that are considered 'general' or 'unspecified' orientation). ${ }^{4}$ Due to limited cell sizes in ISCED levels 3 and 4 in a large number of countries, especially when distinguishing vocational and non-vocational education, we had to aggregate both levels into one. This is as close as possible to the previously tested, ISCED-based "European Survey Version of ISCED” (ES-ISCED) coding scheme (Schneider 2010). For the final coding and the distribution of education categories across countries, see Table 1. We do not report results for cells including fewer than 30 individuals in this table.

The social background of respondents in both cultural and economic ${ }^{5}$ terms is mostly indicated by their parents' education. Parental education is measured with broad ISCED levels only. Three categories can be distinguished: (1) ISCED 2 or below, (2) ISCED 3 and 4 and, (3) ISCED 5 and 6. We have integrated the information from both parents and distinguish whether (1) both parents have ISCED 2 or below (reference category), (2) at least one parent has achieved ISCED 3 or 4, (3) both parents have ISCED 3 or 4, (4) at least one parent has achieved ISCED 5 or 6 or (5) both parents have completed ISCED 5 or 6.

As another variable concerning family cultural background, we include a measure of books in the home when the respondent was 16 years old. Respondents were asked about the number of books in their home based on five different categories ranging from ' 10 books or less' to 'more than 500 books'. We standardized the measure to a mean of 0 and a standard deviation of 1 .

Respondents' migration background is measured by a combination of two indicators: (1) whether a respondent is born abroad and (2) whether his or her native language (mother tongue) is different from the assessment language. The resulting indicator distinguishes whether a respondent has the following status: (1) native-born and native language (reference category), (2) native-born and foreign language, (3) foreign-born and native language and (4) foreign-born and foreign language.

The PIAAC background questionnaire provides three measures of post-educational experiences likely to affect skills and probably partly determined by educational attainment: Firstly, we include a scale indicating whether people read at home ('reading practice'). ${ }^{6}$ The scale was calculated by OECD based on several items on different types of reading activities outside work (e.g. reading of instructions, letters, books, professional journals etc.). It is divided into quintiles, where the lowest category reflects that respondents read never or rarely (e.g. less than once a month) outside work and the highest category that respondents read different types of texts daily or weekly (OECD 2013a, p. 217).

\footnotetext{
${ }_{4}^{4}$ The orientation of the highest qualification is documented in a separate variable in the PIAAC data, which was derived from the national educational attainment questions ex-post. Not all countries seem to have succeeded in this task, resulting in 'unspecified' (Canada and Japan, Denmark, Germany, Sweden, England/Northern Ireland, United States) or missing (Italy and Flanders) orientation, despite the fact that the ISCED mappings indicate an orientation for every educational program. We treated 'unspecified' and missing orientation as non-vocational, i.e. merged it with the genera category, based on the theoretical argument that vocational programs will put less emphasis on the development of basic competencies. For the German data, we used the German Scientific Use File (Rammstedt et al. 2015) in order to derive this information from the country-specific education variable on vocational and higher education.

${ }^{5}$ Parental education is used as a proxy for both cultural and economic resources because there is no measure of parental occupation or wealth available in PIAAC allowing us to differentiate between cultural and economic social background effects.

${ }^{6}$ OECD (2013a) shows that reading outside work has an even stronger relationship with literacy skills than reading at work. Therefore, we have included this scale.
} 


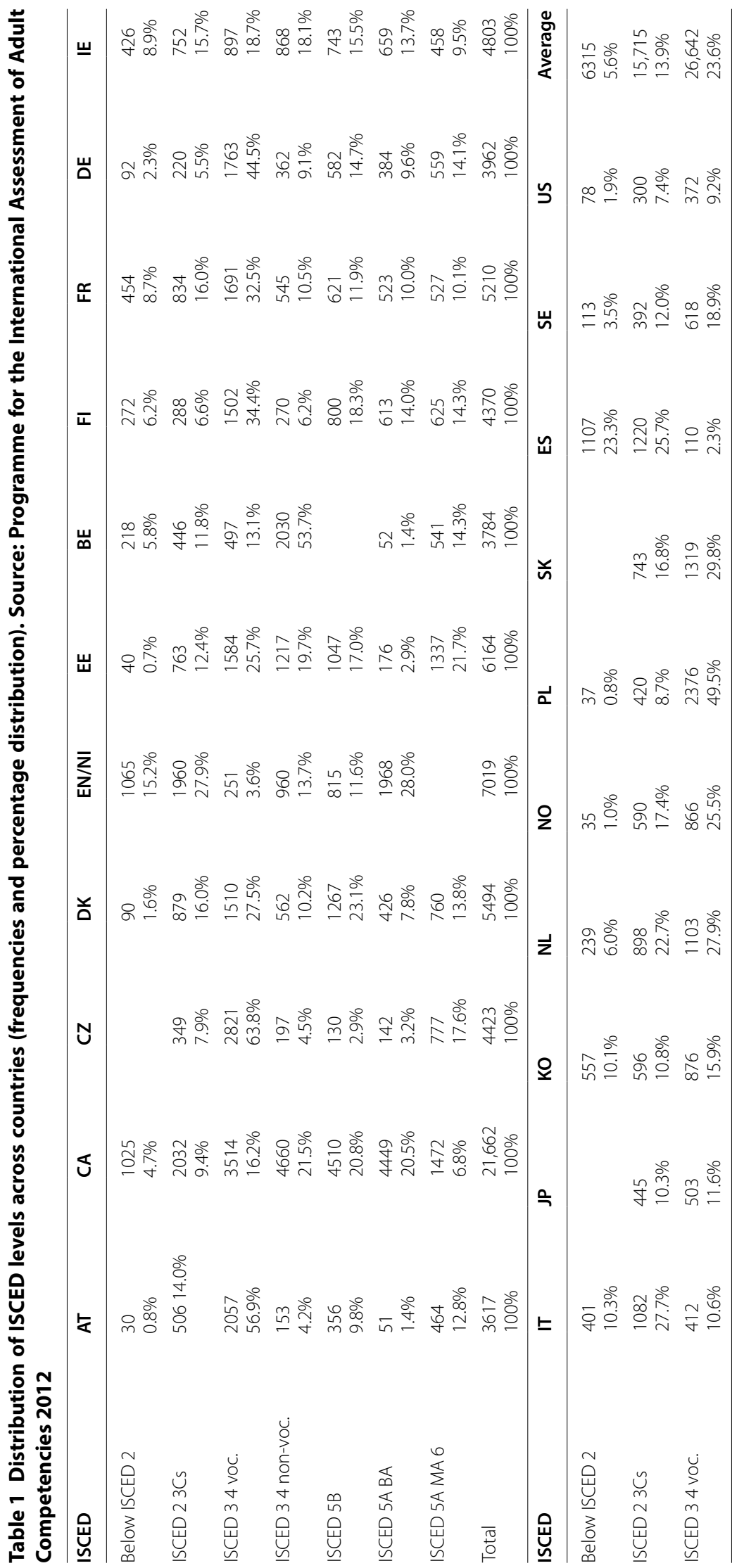




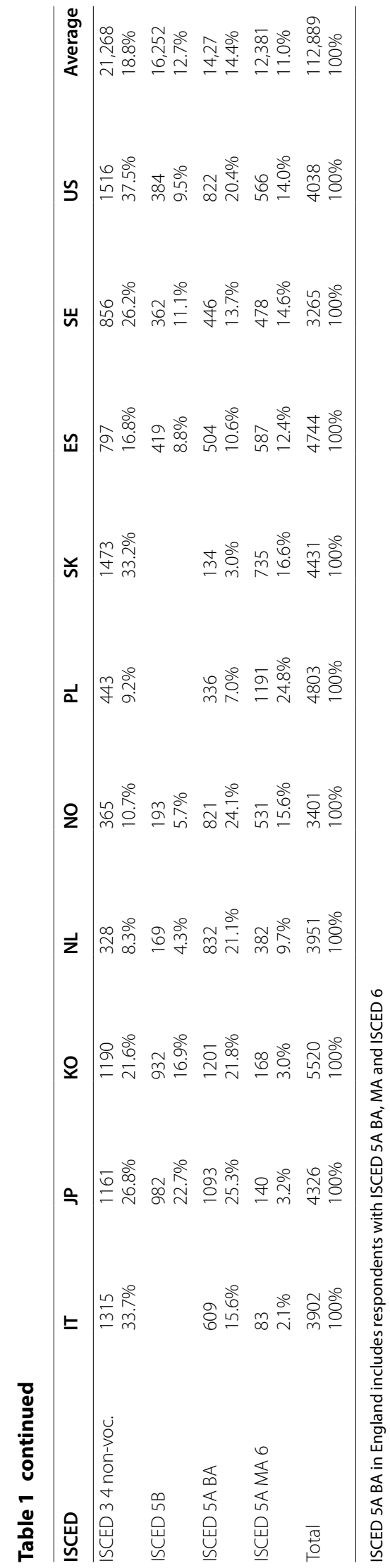


Secondly, we also include participation in formal or non-formal education in the last 12 months. In the following, we refer to this as training activities. Respondents were asked about various different training activities, such as courses and on-the-job training. A variable was created that separated between respondents who had taken part in any activities during the last 12 months or not (reference category). Beyond a direct effect on competencies, these respondents can be expected to have been participating in continuing training in the past.

Thirdly, we include dummy variables indicating occupational group, combining information on employment and an aggregation of major groups (according to skill levels) of the International Standard Classification of Occupations (ISCO) 2008 (International Labour Organisation 2007) and manual/non-manual work. They distinguish whether a respondent is (1) a skilled worker (ISCO major groups 1 to 3, i.e. managers, senior officials, legislators, professionals, technicians and associate professionals), (2) a semiskilled white collar worker (ISCO major groups 4 and 5, i.e. clerks; service workers and shop and market sales workers), (3) a semi-skilled blue collar worker (ISCO major groups 6 to 8, i.e. skilled agricultural and fishery workers; craft and related trades workers; plant and machine operators and assemblers) or (4) an elementary worker (ISCO major group 9) and finally (5) currently not employed (reference category). We thus do not only distinguish whether a respondent is working or not like Park and Kyei (2011), but also take variation in skill use across occupational groups into account (like OECD 2013a; Steedman and McIntosh 2001).

We finally use age and gender as control variables. Ageing may relate negatively with competencies due to decreasing cognitive capacities (Barrett and Riddell 2016; Desjardins and Warnke 2012) and older cohorts have received less formal education (OECD and Statistics Canada 2000a; Shavit and Blossfeld 1993). Also, the specific educational experiences in (in terms of ISCED) equivalent educational programs have changed across cohorts. OECD (2013a) also finds age effects in multivariate models of literacy. As this information is not available as continuous information for all countries, it is categorized into 5-year age bands. Because the effect of age does not seem to be linear (Kirsch et al. 1993), we included dummies for each 5-year band, the dummy for age 25-30 being the reference category. Previous research has shown that there are net gender effects on the different competency domains in some but not many industrialized countries (Desjardins 2003; Maehler et al. 2013; OECD 2011, 2013a, 2015; OECD and Statistics Canada 2000a) so that we also include a dummy variable for gender (male $=0$, female $=1)$.

\section{Analysis method and strategy}

The aim of this paper is to estimate net differences in literacy skill by educational attainment across countries. In order to do so, we run country-wise multiple linear regression models in Stata. Our dependent variable is literacy competency, captured by ten plausible values. The main independent variable is educational attainment, measured as highest educational qualification obtained, coded in detailed ISCED. For the analyses, we use the ado PIAACtools, accounting for the complex sampling structure in PIAAC. ${ }^{7}$

To cross-check our results we also estimated our models using syntax-based programs based on syntax provided by Jan Paul Heisig. We did not find any differences. 
In our first or baseline model, we include respondents' detailed educational attainment as independent variable only. This model thus estimates the gross (unadjusted) relationship between formal education and competencies. The results of this model will likely overestimate the effect of education on adult competencies because educational attainment and competencies are both to some degree caused by two common third variables, family conditions and cognitive ability (confounding bias, see for example Elwert and Winship 2013). In the second model, we thus introduce variables measuring family conditions, namely parental education, migration background and books in the home at age 16. This allows us to estimate the relationship between educational attainment and competencies net of family background. We also control for age and gender in this model.

Even then, this model may still overestimate the direct impact of formal education on skill because so far omitted variables that are related to educational attainment may affect skills rather than educational attainment itself. In our third model, we thus finally include variables affecting skills after completion of formal educational, such as occupational group, reading at home and participation in training activities. Theoretically, these variables are considered to lie on the causal path between educational attainment and adult competencies so they may explain to some degree why educational attainment positively relates to literacy skills, or why the relationship between education and literacy differs across countries. The resulting residual education-skill relationship is thus the remaining direct relationship, not total relationship, between education and skills, which cannot be explained by either family background or post-school skill development. ${ }^{8}$

\section{Results}

We start out by describing the unadjusted results regarding the gross relationship between educational qualifications and skills (Model 1, see Table 3 in appendix for more details), and then turn to the adjusted regression models, first describing differences compared to the unadjusted model when adjusting for antecedents of education and skills (Model 2, see Table 4 in appendix for more details), and second describing further differences when also adjusting for post-education factors of skill development (Model 3, see Table 5 in appendix for more details).

Figure 1 shows average literacy scores by detailed education categories for all PIAAC countries (basically, conditional means) resulting from Model 1. According to this model, the different ISCED categories follow the same order in most countries, meaning that higher level educational qualifications are associated with higher literacy skills, and within ISCED levels, non-vocational education is usually associated with higher competencies than vocational education. For example, in all countries, respondents with less than lower secondary education have lower average literacy scores than respondents with completed lower secondary education, and those in turn have lower average scores than respondents with completed vocational and non-vocational upper secondary

\footnotetext{
${ }^{8}$ We distinguish models 2 and 3 for two reasons: firstly, because we want to estimate the total net relationship between education and skills, which is achieved by model 2, where entering variables on the causal path from education to skills (like in model 3) would introduce overcontrol bias (Elwert and Winship 2013). Secondly, model 3 is, like many approaches trying to disentangle direct and indirect 'effects', at risk of endogenous selection bias (Elwert and Winship 2013): rather than conceptualizing occupation, reading habits and adult learning as a mediator between formal education and skills, they could also be regarded as common outcomes (descendants) of education and skills. In this alternative theoretical model, they would be collider variables and controlling for them would introduce a spurious association between education and skills. Still we consider the estimation of model 3 worthwhile in order to control for compositional effects regarding post-educational experiences between countries.
} 


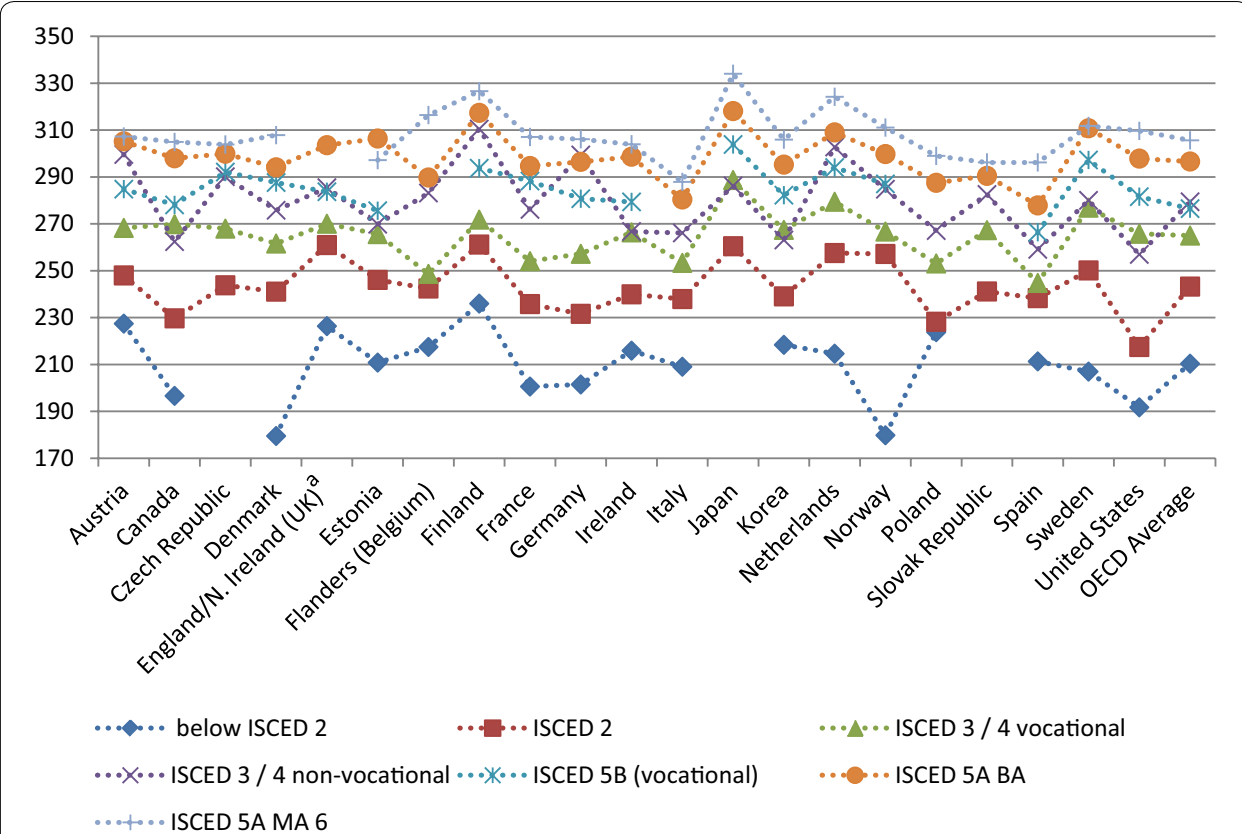

Fig. 1 Literacy skills by detailed educational attainment and country, unadjusted. Source: Programme for the International Assessment of Adult Competencies (PIAAC), 2012; own calculations. Notes ISCED = International Standard Classification of Education. Sample is restricted to adults aged 25-65 years in 2011 and to respondents who completed their highest educational qualification in the country they participated in for PIAAC. ${ }^{a}$ ISCED 5A BA for England/Northern Ireland includes both BA and MA, as well as ISCED 6

education. Within tertiary education, we find considerable differences between qualifications from short programs that are vocationally oriented (ISCED 5B, usually $2-3$ years duration full-time) and academic degrees at Bachelor level (ISCED 5A medium) for all countries in which qualifications classified as ISCED 5B exist.

However, there are some remarkable exceptions to the hierarchical ordering of average literacy skills by education category, mostly affecting upper secondary and vocational tertiary education. The first one are respondents with ISCED 3 or 4 non-vocational in comparison to respondents with vocational tertiary education (ISCED 5B): the former group achieves significantly higher or at least comparable (average) competency scores than the latter group in several countries (especially Austria, Finland, the Netherlands and Germany). However vocational tertiary qualifications consistently go along with higher competencies than vocational upper secondary ones. Another exception is that in Germany, non-vocational upper secondary graduates even slightly (and significantly) outperform tertiary graduates with degrees from medium-duration $5 \mathrm{~A}$ (i.e. academic) programs-a group that is comparatively small though and potentially negatively selected due to Bachelor's degrees having been introduced in Germany only recently. ${ }^{9}$ Finally, in Estonia, graduates from long (Master's level) academic tertiary programs show 9 points lower literacy scores than those from medium (Bachelor's level) duration programs. Here again, the Bachelor's level category is rather small though.

\footnotetext{
9 The common pre-Bologna qualification from polytechnic higher education ('Diplom Fachhochschule') should have been classified here, too, but individuals with this qualification are included in the ISCED 5A long category because the measurement instrument used in PIAAC does not differentiate them from university graduates.
} 
In some cases, while the order is not broken, there are only very small competency differences between categories located at different main ISCED levels: respondents in Flanders and Spain with ISCED 3 or 4 vocational in comparison to ISCED 2 or 3C short only achieve average literacy levels which are 7 points higher. The difference of mean competency scores between respondents with ISCED 3 or 4 non-vocational and qualifications from vocational tertiary education (ISCED 5B) is small in the Czech Republic, England/ Northern Ireland, Norway and Estonia, and the difference between the former and those with medium-duration, i.e. Bachelor's level, university programs (ISCED 5A) is small in Austria, Flanders, Finland and the Netherlands.

Looking more closely at vocational and non-vocational qualifications at ISCED levels 3 and 4, we find substantial literacy skill differences in two-thirds of the countries. In total, in ten out of 21 countries, respondents with non-vocational education show a statistically significantly higher average literacy score than respondents with vocational education. The highest differences can be found in Germany and Finland, where nonvocational upper secondary education is associated with average literacy scores that are 42 or respectively 39 points higher than vocational education. While respondents with vocational upper secondary education on average score slightly higher than respondents with non-vocational education at this level in the United States and Canada (9 and 8 points respectively), these differences are not statistically significant. The within-upper secondary competency differences in Estonia, Ireland, Japan and Korea seems to be almost negligible and are statistically not significant in Estonia and Ireland.

Turning to differences between countries within detailed education categories, in all educational groups, we see a range of about 40-60 competency scores, i.e. about one competency level, between the countries with the highest average competency scores and the lowest average scores in all education groups. The range is especially high in the lowest educational group, i.e. for respondents who have no educational qualification or a qualification below ISCED level 2 (ranging from average competency scores of 179 in Denmark to 236 in Finland). In most countries, the group of low educated adults is very small, however, accounting for only $5.6 \%$ of all respondents across countries (see Table 1). With respect to completed lower secondary education, we find somewhat smaller competency differences of around 45 points between countries. Finland, Japan and England/Northern Ireland ${ }^{10}$ score highest in this group with around 260 points and the United States the lowest with 217 points. Looking at the completion of vocational upper and post-secondary education (ISCED 3 and 4) we find similar cross-national differences (a difference of 44 points between Japan with 289 and Spain with 245 points). The cross-country range is, with 53 points, rather large again for non-vocational upper and post-secondary education (United States: 257, Finland: 310). For vocational tertiary, Bachelor and Master level education the ranges are 37, 46 and 46 points respectively.

\footnotetext{
10 This result is different from what is usually found for the UK, because we reclassified all respondents with GCSEs, the main general school leaving qualification at age 16 which is required to proceed to A-Levels, which in turn give access to university studies, to ISCED level 2. In OECD statistics, only those with 'weak' GCSEs (less than 5, or grades lower then C) are classified at ISCED level 2. Our reason for doing so is that other countries classify such programs at ISCED level 2, and this is in better accordance with ISCED criteria. While ISCED category 3C was never meant to be used for general educational programs, the UK classifies their GCSEs at ISCED 3C if the result is 'strong' (5 or more at grades A to C). Using the official ISCED mapping for the UK, the competency levels at lower and upper secondary education in England/Northern Ireland would be much lower. Unfortunately, the international organizations have only very limited influence on how countries assign educational programs and qualifications to ISCED, which opens the door to politically motivated classification decisions.
} 
This also means that respondents with more education score lower on the literacy scale in some countries than respondents with less education in other countries. As an example, respondents with ISCED 3 or 4 non-vocational in Finland score about one competency level above respondents with ISCED 5B in Spain. Therefore, in terms of literacy skills, ISCED categories are neither substantively comparable nor consistently ordered across countries, at least when looking at the unadjusted means.

In terms of overall strength of the association as measured by explained variance in this bivariate model, we can see that it also varies considerably between countries: The adjusted $\mathrm{R}^{2}$ for the Estonia is 0.15 , for the Czech Republic 0.18 and for Austria 0.19 , while the value is 0.32 for Flanders, and 0.34 for France and the Netherlands (see Table 2). Education and literacy competency thus seem to be more closely related in the latter countries than in the former, but in all countries the association is far from perfect.

When looking at the results of model 2 adjusting for age, gender, parental education, migration background and books in the home at age 16 (Fig. 2), the hierarchy of skills between ISCED levels remains mostly the same as in model 1. The negative literacy gap between respondents with ISCED 3 or 4 non-vocational and respondents with ISCED $5 \mathrm{~B}$ has substantially decreased in all countries with the exception of Germany and the

Table 2 Explained variance (adjusted $R^{2}$ ) for three models. Source: Programme for the International Assessment of Adult Competencies 2012

\begin{tabular}{|c|c|c|c|c|c|}
\hline & \multicolumn{3}{|c|}{ Adjusted $\mathrm{R}^{2}$} & \multicolumn{2}{|c|}{$\begin{array}{l}\text { Increment to } R^{2} \\
\text { from model } 1 \text { to }\end{array}$} \\
\hline & Model 1 & Model 2 & Model 3 & Model 2 & Model 3 \\
\hline Austria & 0.19 & 0.30 & 0.33 & 0.11 & 0.03 \\
\hline Canada & 0.23 & 0.36 & 0.41 & 0.13 & 0.05 \\
\hline Czech Republic & 0.18 & 0.23 & 0.26 & 0.05 & 0.03 \\
\hline Denmark & 0.24 & 0.38 & 0.42 & 0.13 & 0.04 \\
\hline England/N. Ireland (UK) & 0.23 & 0.33 & 0.37 & 0.10 & 0.04 \\
\hline Estonia & 0.15 & 0.24 & 0.26 & 0.09 & 0.02 \\
\hline Finland & 0.28 & 0.40 & 0.43 & 0.12 & 0.03 \\
\hline Flanders (Belgium) & 0.32 & 0.41 & 0.43 & 0.09 & 0.02 \\
\hline France & 0.34 & 0.41 & 0.43 & 0.07 & 0.02 \\
\hline Germany & 0.28 & 0.39 & 0.42 & 0.10 & 0.04 \\
\hline Ireland & 0.28 & 0.35 & 0.37 & 0.07 & 0.02 \\
\hline Italy & 0.24 & 0.29 & 0.31 & 0.05 & 0.02 \\
\hline Japan & 0.22 & 0.32 & 0.33 & 0.10 & 0.01 \\
\hline Korea & 0.31 & 0.37 & 0.39 & 0.06 & 0.02 \\
\hline Netherlands & 0.34 & 0.48 & 0.50 & 0.13 & 0.02 \\
\hline Norway & 0.23 & 0.40 & 0.43 & 0.17 & 0.03 \\
\hline Poland & 0.22 & 0.26 & 0.28 & 0.04 & 0.02 \\
\hline Slovak Republic & 0.22 & 0.26 & 0.27 & 0.04 & 0.01 \\
\hline Spain & 0.31 & 0.39 & 0.41 & 0.09 & 0.02 \\
\hline Sweden & 0.24 & 0.47 & 0.50 & 0.23 & 0.03 \\
\hline United States & 0.31 & 0.42 & 0.44 & 0.10 & 0.03 \\
\hline OECD average & 0.26 & 0.35 & 0.38 & 0.10 & 0.03 \\
\hline
\end{tabular}

Sample is restricted to adults aged 25-65 years in 2011 and to respondents who completed their highest educational qualification in the country they participated in for PIAAC. Model 1 includes literacy skills by detailed educational attainment. Model 2 adjust for age, gender, parental education, migration background and books in the home at age 16. Model 3 additionally adjusts for occupational group, reading at home, and participation in training activity 


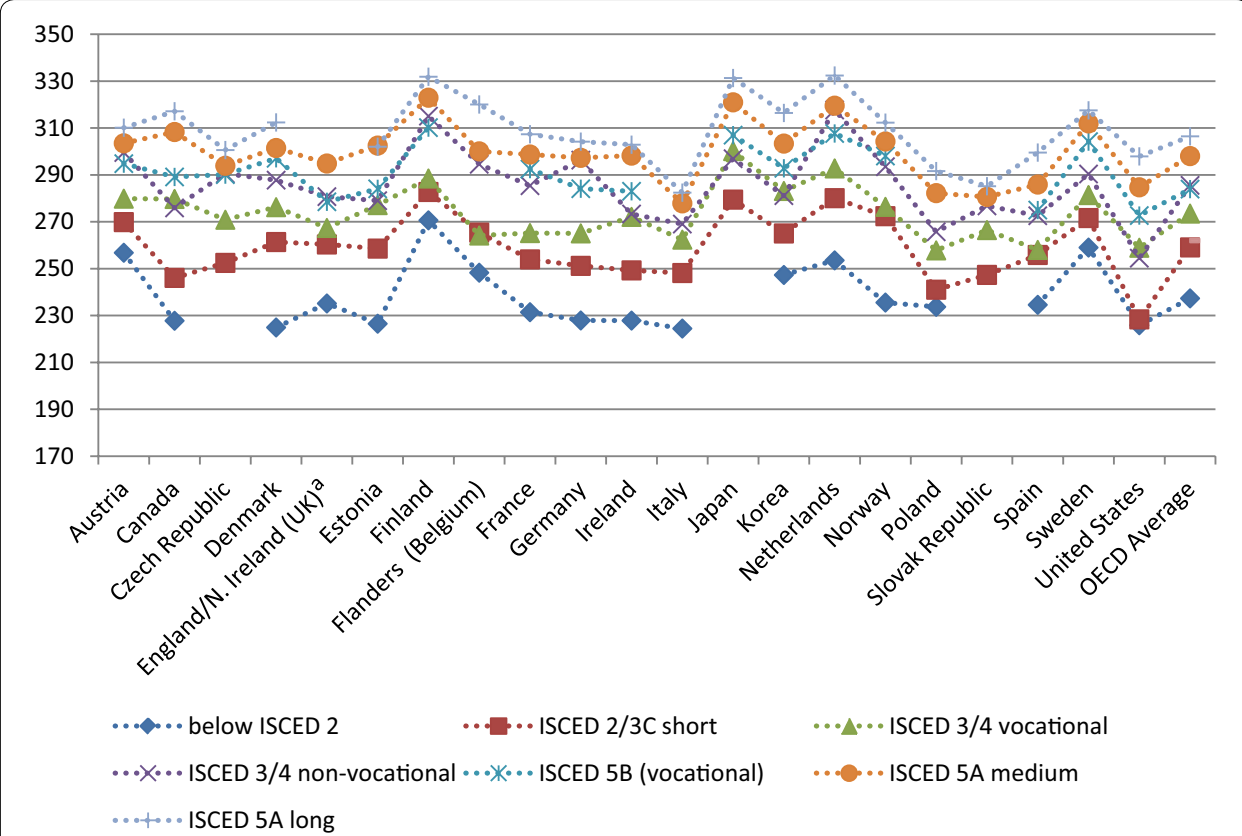

Fig. 2 Literacy skills by detailed educational attainment and country, adjusting for age, gender, parental education, migration background and books in the home at age 16. Source: Programme for the International Assessment of Adult Competencies (PIAAC), 2012; own calculations. Notes ISCED = International Standard Classification of Education. Sample is restricted to adults aged 25-65 years in 2011 and to respondents who completed their highest educational qualification in the country they participated in for PIAAC. Reference categories: 25-30 years, male, both parents ISCED 2 or below, no migration background, standardized measure of books. ${ }^{a}$ ISCED 5A BA for England/Northern Ireland includes both BA and MA, as well as ISCED 6

Netherlands, however. When comparing vocational and non-vocational qualifications at ISCED level 3 and 4 while adjusting for various background variables, we still find lower competencies for vocational qualifications in ten countries. However, the gap between these qualifications has diminished in all countries but the Netherlands, especially in Germany and Finland, and to a lesser extent in Austria. The difference is still significant, and in Germany and Finland they remain substantial. Japan, Korea, Ireland and Estonia join Canada and the United States in vocational upper secondary education leading to the same or even slightly higher literacy skills as non-vocational upper secondary education. Turning to the differences between vocational and general/academic education at tertiary level, we find that also in model 2 respondents with a general education score higher in literacy than respondents with a vocational qualification in all countries. Similar as for ISCED level 3 and 4, the gap between qualifications classified as ISCED 5A Bachelor level and ISCED 5B is lower now than in model 1. Especially in Estonia, Finland and Austria, this gap diminished after the adjustment but especially in the former two countries it remains quite substantial and significant. In summary, while some of the relationship between educational attainment and literacy skills is due to social background and migration/language status, formal education still makes a large difference for the achievement of adult literacy skills, whether because of differential skill selectivity or differential skill acquisition in different programs. 
In Model 2, the competency gap between respondents with high and low educational qualifications is smaller than in the unadjusted model. However, as in the unadjusted model, there are still large competency differences between respondents with 'equivalent' educational qualifications across countries. Only for respondents below ISCED level 2, the differences across countries diminish between model 1 and model 2. For lower secondary or non-vocational upper secondary education and, to a lesser extent, for the different qualifications at the tertiary level, the differences between the country with the highest and the lowest average proficiency even increase: in the Netherlands, respondents at level 3 or 4 vocational achieve 318 points, while in the United States respondents achieve 254 points-a competency gap of more than half a competency level and 10 points more than in model 1 . So while adjusting for important antecedents of both educational attainment and skills reveals that the low levels of literacy of the low educated are to a large extent explained by social and migration background, differences across countries in composition by social and migration background do not make crosscountry differences in literacy skills for comparable education categories disappear-on the contrary. Also the countries are ordered more similarly across education categories in terms of average literacy skills than in model 1, with Finland, the Netherlands and Japan always amongst the top and Italy, the Slovak Republic and the United States always amongst the bottom performers.

Between models 1 and 2, the adjusted $\mathrm{R}^{2}$ has increased by almost 10\% (from 26 to $35 \%)$ on average across countries. The strongest increase can be seen for Sweden, where the adjusted $\mathrm{R}^{2}$ for model 2 is $47 \%$, up from $24 \%$ in the unadjusted Model 1 (see Table 2). This may be due to the Swedish educational system not being very selective, an education policy measure to counter social inequalities in education, but skill development still strongly depending on family background. Therefore, formal education is not as strongly a mediator of social background effects on skills in Sweden as it is in other countries.

In model 3, we have introduced further adjustments, namely variables which are likely to affect literacy skills after initial education (Fig. 3). These were occupational group, reading at home, and participation in training activities in the last 12 months. After introducing these variables, the general patterns we already saw in models 1 and 2 remain the same. We will only highlight the most important differences. In model 3 the skill differences between different educational groups become even less distinct within each country, this time specifically in the top education categories: the highly educated have substantially better opportunities for further developing their literacy skill in their working lives than the lower educated, and they also read more in their leisure time. However, we can still find considerable differences in literacy skills between respondents in the same ISCED category across countries.

In comparison to models 1 and 2, we see that the hierarchy of educational levels is less obvious. In particular, this concerns the differences between non-vocational ISCED 3 and 4 qualifications and qualifications at ISCED level 5B. As in model 1 and 2 , in some countries, respondents with ISCED 3 or 4 non-vocational score higher than respondents with ISCED 5B. What has changed, however, is the gap between these two which became smaller in all countries. It can now be observed in several countries that respondents with lower secondary education (ISCED level 2 or $3 \mathrm{C}$ short) score higher 


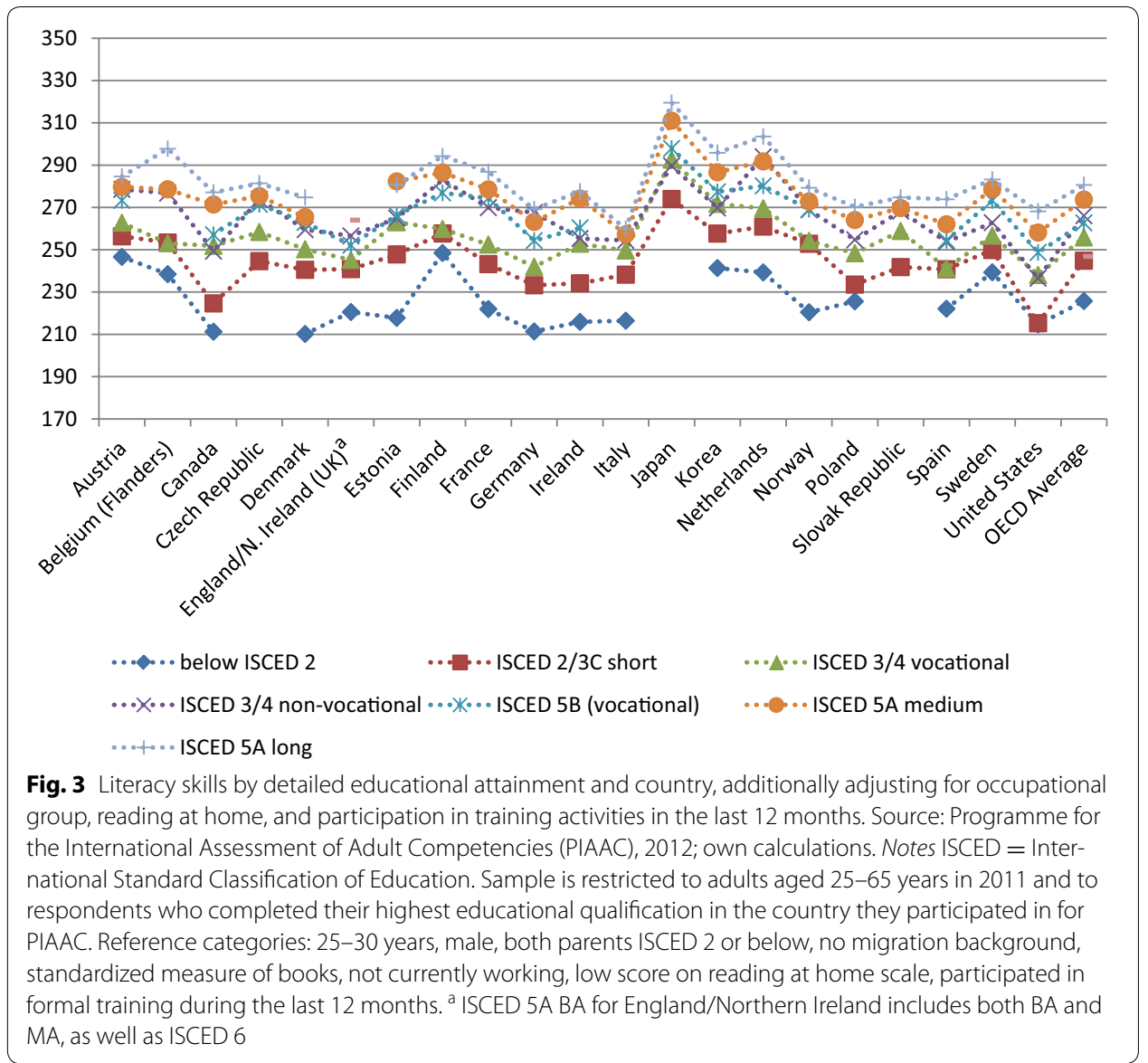

than respondents with ISCED level 3/4 vocational (Flanders, England/Northern Ireland, Finland and Norway). This hints at the literacy skill advantage of those with vocational upper secondary education compared to those with lower secondary education in model 2 being due to their more favorable labor market placement and reading habits rather than their vocational upper secondary education itself (however, their labor market placement to some degree depends on it obviously). Furthermore, we also find in model 3 that literacy scores of respondents with ISCED level 3 or 4 non-vocational do not differ much from scores of respondents with Bachelor level education (with the exception of Canada, Estonia, Ireland, Japan, Korea, Sweden and the United States).

Comparing fully adjusted average literacy by ISCED levels across countries, we see that the gap between countries scoring the highest and scoring the lowest has diminished for the lowest educational group but has increased for ISCED levels 3 and above. For respondents below ISCED 2, the score is 239 for Sweden and 210 for Denmark, a competency gap of a bit more than half a competency level. It was 56 points in Model 1 . At ISCED 5B, the competency gap between countries increased from 37 points (between Japan with 304 and 266 in Spain) to 49 points (between Japan with 298 and Spain with 249). Altogether, even after adjusting for a wide range of factors, there are still substantial differences in average literacy skills between countries for supposedly comparable education categories. 
The adjusted $R^{2}$ suggests that with model 3 not more variance in skills can be explained in all countries, in contrast to model 2 . This suggests that the background variables in model 2 seem to be more important in explaining the variation in literacy skills. Introducing the additional mediating variables in model 3 does not add explanatory power to the model. However, since the effects of education on skills somewhat decrease between models 2 and 3 in most countries, those additional variables mediate some of the effects of educational attainment on skills so that model 3 can be interpreted as showing the relationship between educational attainment and literacy skills 'net' of labor market experiences and cross-country differences therein.

\section{Summary and discussion}

We find considerable differences across countries in the average literacy skills associated with supposedly equivalent education levels, as well as in the strength of association of educational qualifications and skills. Our results suggest that some of these differences are due to differences across populations in characteristics that influence education and skill acquisition before achieving educational qualifications, such as family background, as well as experiences that occur after the completion of educational qualifications, such as daily reading practices and the job situation. However, even after adjusting for a wide range of correlates of education and literacy skills, substantial cross-country differences in average skills within education categories remain-and in some cases even become stronger. In contrast to Park and Kyei (2011), we do not find that the differences between countries are smaller at higher education levels than at lower education levels, which may be due to our more comprehensive set of controls as well as a broader set of countries covered in PIAAC than in IALS.

Furthermore, confirming results by Maehler et al. (2013) for Germany on an international scale, we find substantial heterogeneity in literacy within broad education levels across countries. This shows that it is in fact worth looking at detailed education categories rather than just broad heterogeneous levels. The cross-country differences in skills by detailed education categories seem to be related to characteristics of the respective educational systems: In those countries where there are no substantial skill differences between vocational and non-vocational qualifications at the upper secondary level (Canada, Estonia, Ireland, Japan, Korea and the United States), vocational education is not very vocationally specific, which may mean that in such 'pseudo-vocational' programs literacy competencies are improved as much as in general programs. Another potential explanation, however, is that the results reflect sorting and educational choices by competency: In the above countries, skill selectivity may not differ between (pseudo-) vocational and non-vocational programs. In contrast, in countries with a strong vocational upper secondary system, such as Germany and Finland, people who initially have a higher literacy competency follow more general tracks while people with lower competencies engage in vocationally oriented programs.

This puts the validity of broad education levels as proxies for general skills into considerable doubt: In many countries, specifically those with distinct vocational training systems, graduates of vocational education and training have substantially lower literacy skills than graduates of non-vocational education at both the upper secondary and tertiary levels. Literacy skills are usually analyzed and reported in only three broad 
education levels (low, medium and high) and our results suggest that average literacy scores by broad education level for any given country seem to dependent to a large degree on the prevalence of the vocationally educated groups within those levels.

Available comparative research on differences across countries in adult competencies for comparable education groups concentrate on differences in the organization of or resource inequality within educational systems (Heisig and Solga 2015; Park and Kyei 2011). Another explanation concerns differences between countries in the selectivity of specific educational categories. We cannot tell whether the skill differences that we find within broad education levels can be explained by selection effects or skill acquisition effects. This is due to the fact that variables such as prior learning experiences, cognitive ability and-relevant for differences at tertiary level-literacy skills at completion of secondary education cannot be accounted for with PIAAC.

There is, however, also an interpretation for these results that concerns the methodology of PIAAC, and specifically the measurement of educational attainment using ISCED. The ISCED classification criteria (UNESCO Institute for Statistics 2006), which are admittedly proxy-criteria due to lacking direct indicators, may be ill-suited to capture the actual complexity of content of educational programs, the concept ISCED intends to measure. The complexity of content of an educational program should theoretically be quite strongly related to the average literacy skills that completers of the program show, because literacy skills highly correlate with other types of general skills. The most important classification criteria defined by ISCED are typical age of entry into an educational program and theoretical program duration, together forming the cumulative duration of education at the end of the program. Sometimes additionally a minimum entry requirement in terms of a level and/or type of program previously completed, or the level and/or type of program the program to be classified is designed to prepare for, are also defined. Obviously, these criteria exclusively refer to the structure of educational systems, not to the complexity of content and related demand placed on learners or even skill outcomes. In fact, we are not aware of any study evaluating the extent to which the ISCED criteria do capture complexity of content. Our results make us skeptical in this regard: Even though ISCED offers the tools to distinguish between general and vocational education, for the same duration of education, equal complexity of content is assumed for vocational and general programs, and thus they are assigned to the same main ISCED level. Our research however suggests that in terms of literacy, the complexity of content of vocational programs may be substantially lower, so they more strongly draw in participants from the lower end of the skill distribution at the completion of the previous level, especially in countries with highly occupationally specific vocational training. The current ISCED criteria seem, on their own, incomplete to well differentiate educational programs by their degree of complexity of content.

Limitations of the study are similar to those of previous studies using IALS, ALL or NALS data, because by and large, these surveys share some design weaknesses (see also Desjardins 2003; Kerckhoff et al. 2001; Park and Kyei 2011): incomplete measures of family conditions and post-school experiences as well as the absence of a measure of generalized cognitive ability or literacy skills at earlier time points lead to residual confounding, so that data better describing learning contexts during childhood, youth and adulthood would improve the interpretability of results. Because these variables are 
not measured in PIAAC, the estimation of the net effect of level of education on literacy skills is problematic, as the influences of theses variables cannot fully be accounted for. Therefore the education effect is likely still overestimated in models 2 and 3 . in the absence of panel (let alone experimental) data, it is impossible to correctly model causal relationships between formal education, adult competencies, and their mediators such as employment, occupation, adult training or reading practice and thus better understand the skill formation process and make public policy recommendations (Raudenbush and Kim 2002). Basically, research based on cross-sectional surveys such as PIAAC cannot differentiate between the theoretically equally plausible causal mechanisms of literacy selection (i.e. students with higher literacy progressing further or to different types of programs in formal education) and literacy development (i.e. formal education producing higher literacy) distinguished by Reder (1998). This is especially relevant for the differences between vocational and non-vocational upper secondary education, as well as results at the tertiary level.

Another issue that needs to be considered when interpreting our results are differences in 'literacy related nonresponse' (LRNR) across countries. The number of literacy related non-respondents ranges between 0\% (Finland, Poland and Sweden) and 5.2\% (Flanders) in our sample (OECD 2013a). Van de Kerckhove et al. (2013) show that a LRNR share of $2 \%$ has little impact on the overall score but that significant bias can be introduced with a share of $8 \%$ LRNR. This needs to be considered when interpreting the results of our analyses as it can be assumed that literacy related non-response is related to lower literacy skills in the interview language (Van de Kerckhove et al. 2013). This means that countries with a higher share of LRNR are likely to have lower literacy skills than reported. Furthermore, it is likely that LRNR occurs more often in lower educational groups in most countries.

\section{Conclusions}

We would like to offer two kinds of conclusions: one for researchers trying to proxy competencies with information on educational attainment, and one for future PIAAC studies. With respect to the first issue, looking at detailed ISCED categories reveals skill similarities across and differences within ISCED main levels, which means that for analyzing skills, ISCED levels show a low degree of validity. Therefore, analysts trying to use educational attainment data to proxy differences between individuals in literacy (or other general basic) skills should not use ISCED main (or even broad) levels, but rather code detailed education categories according to their competency outcomes. This means that individuals with qualifications from vocational tertiary education should be aggregated with individuals with non-vocational upper secondary education (ideally ISCED level 4 only) rather than with individuals with academic tertiary qualifications, as is usually done, or, better still, be kept separate. Furthermore, given the strong differences between vocational and non-vocational upper secondary education in a large number of countries covered in PIAAC, these two categories should also be coded separately whenever possible, at least for those countries where skill differences are large. In many 
countries, the average competencies of the vocationally educated are closer to those of individuals with lower secondary education (ISCED level 2) than to those of individuals with non-vocational upper secondary education. Basically, when proxying competencies, in countries with strong vocational training systems the vocationally educated should, be downgraded to the next lower ISCED level. ${ }^{11}$

Regarding recommendations for PIAAC, there are several points to make. Firstly, despite the fact that formal education is undisputedly the most important context of skill formation, educational attainment is treated in adult literacy surveys such as PIAAC as a mere 'background variable'. As a consequence, it is not as well measured as one might wish: For example, qualifications resulting from vocational and general programs, or between those preparing primarily for university and those preparing primarily for the labor market, are not easily distinguished even though these differences can be expected to be important for literacy skill formation. The variable on orientation was not specified ex-ante, apparently leading to ex-post coding problems for many countries. As another example, the differentiation between the Bachelor's and Master's level cannot be drawn in all countries due to limitations of the measurement instruments. In the UK, it cannot be drawn at all, and in Germany, there is a large element of misclassification in these categories of the variable. Therefore, we would strongly recommend (1) to give the relationship between educational attainment, basic skills and labor market outcomes more theoretical thought and thus specify more relevant and valid harmonized target variables, and (2) to put more quality control into place regarding the ex-ante outputharmonization of educational attainment in any future PIAAC cycle (regarding the harmonization of education in comparative surveys, see e.g. Schneider 2010; Schneider et al. 2016; Wolf et al. 2016).

Secondly, we do not know anything about the pathway an individual has taken through the educational system, i.e. how the highest qualification that is measured was achieved. Different pathways, especially in countries where multiple options are available at every transition point, are likely to provide different access barriers and learning environments, and thus result in different literacy skills. Fortunately, OECD is already investigating these issues for the upcoming PIAAC cycle. Thirdly, we would strongly suggest enriching the set of background variables to be more able to tease out different causal mechanisms concerning adult skill development and avoid conflating many different effects in the measure of parental education. Without going full-scale longitudinal, causal modeling more strictly speaking will of course remain impossible.

Finally, we would like to offer some ideas for further research: Firstly, it would be worthwhile to extend this study by also including the nine PIAAC round 2 countries for which data were collected in 2014. Given these countries are less developed than round 1 countries, we would expect to find even more variation in literacy skills by educational attainment. Secondly, we have ignored potential interaction effects in this study in order not to overcomplicate the models. Most importantly, it is quite plausible that the relationship between education and literacy skills changes across cohorts, mostly because younger generations have benefited from educational expansion (Shavit and Blossfeld 1993) and formal education can be expected to be more relevant to the skills of younger

11 This does not imply that vocational education is generally less valuable than general education-only that, in terms of literacy skill outcomes, it is not comparable to general education at the same level of education. 
individuals just because they have left education more recently. Thirdly, one could try to systematically scale educational attainment by directly assessed skills across countries to develop more comparable measures of skills, based on information on educational attainment coded in ISCED only, which could then also be applied to other data than PIAAC. One could also use PIAAC data for benchmarking specific ISCED categories for specific age groups across countries, following the approach taken by. Finally, the obvious next step in substantive analysis would be to investigate contextual effects on cross-country differences in competencies at given education levels or gaps between specific education levels, building on prior research by Park and Kyei (2011) and Heisig and Solga (2015). In our view it is important to learn more about the individual determinants of adult skills and how these differ across countries, since this could provide us with important lessons for the future: it is very clear that adults to a large extent transmit their competencies to their children in most if not all countries. Because of data constraints, prior sociological research has largely focused on inequality of educational opportunity in terms of educational attainment (for a review, see Breen and Jonsson 2005). With PIAAC data, as limited as they may be in terms of background measures, it is possible to add to this the study of social inequality in competencies across countries.

Authors' contribution

NM and SLS participated in the rationale of the paper. NM carried out the coding and statistical analyses and prepared the tables and graphs. SLS carried out the literature review and theoretical work. NM and SLS both drafted the manuscript. Both authors read and approved the final manuscript.

Competing interests

The authors declare that they have no competing interests.

Availability of data and materials

The datasets supporting the conclusions of this article are available on the OECD website (http://www.oecd.org/skills/ piaac/publicdataandanalysis.htm) and at the GESIS data archive (doi:10.4232/1.12385 at https://dbk.gesis.org/dbksearch/ sdesc2.asp?no=5845).

\section{Appendix}

See Tables 3, 4 and 5 . 


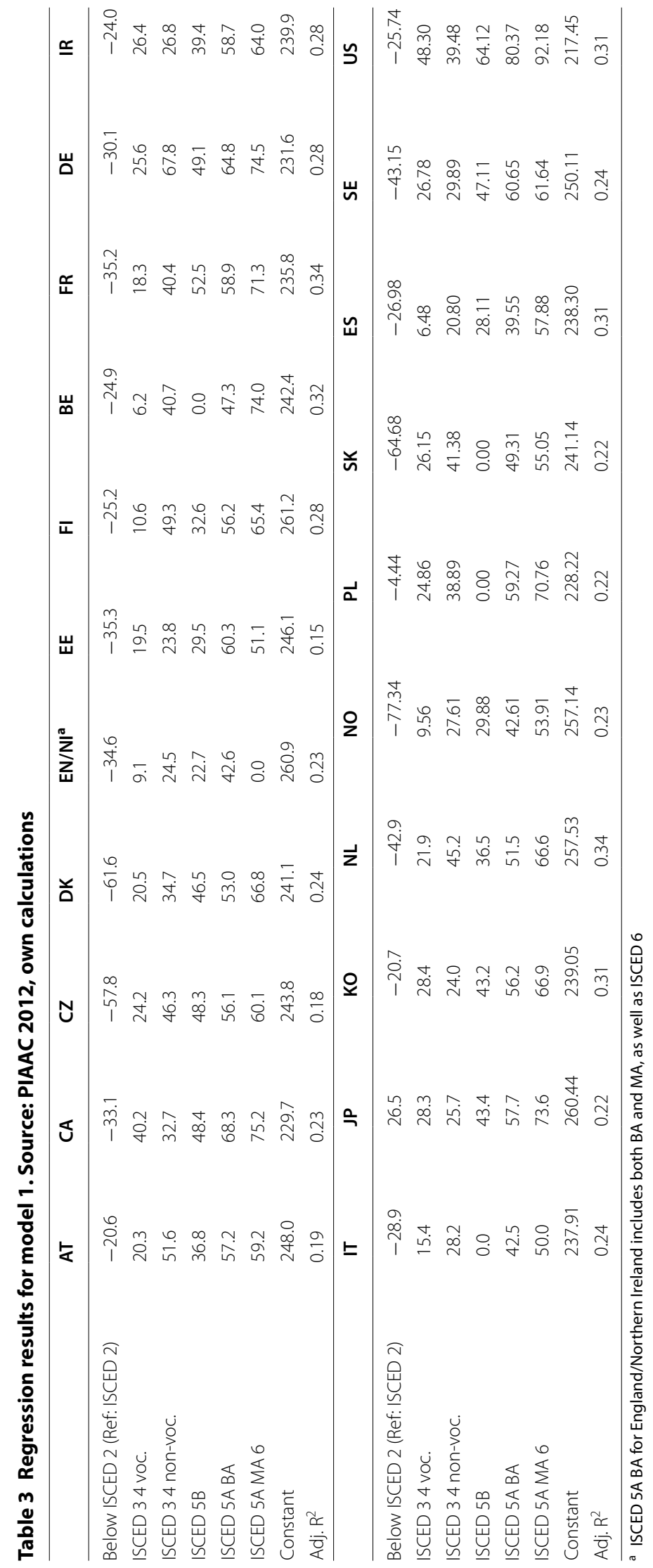




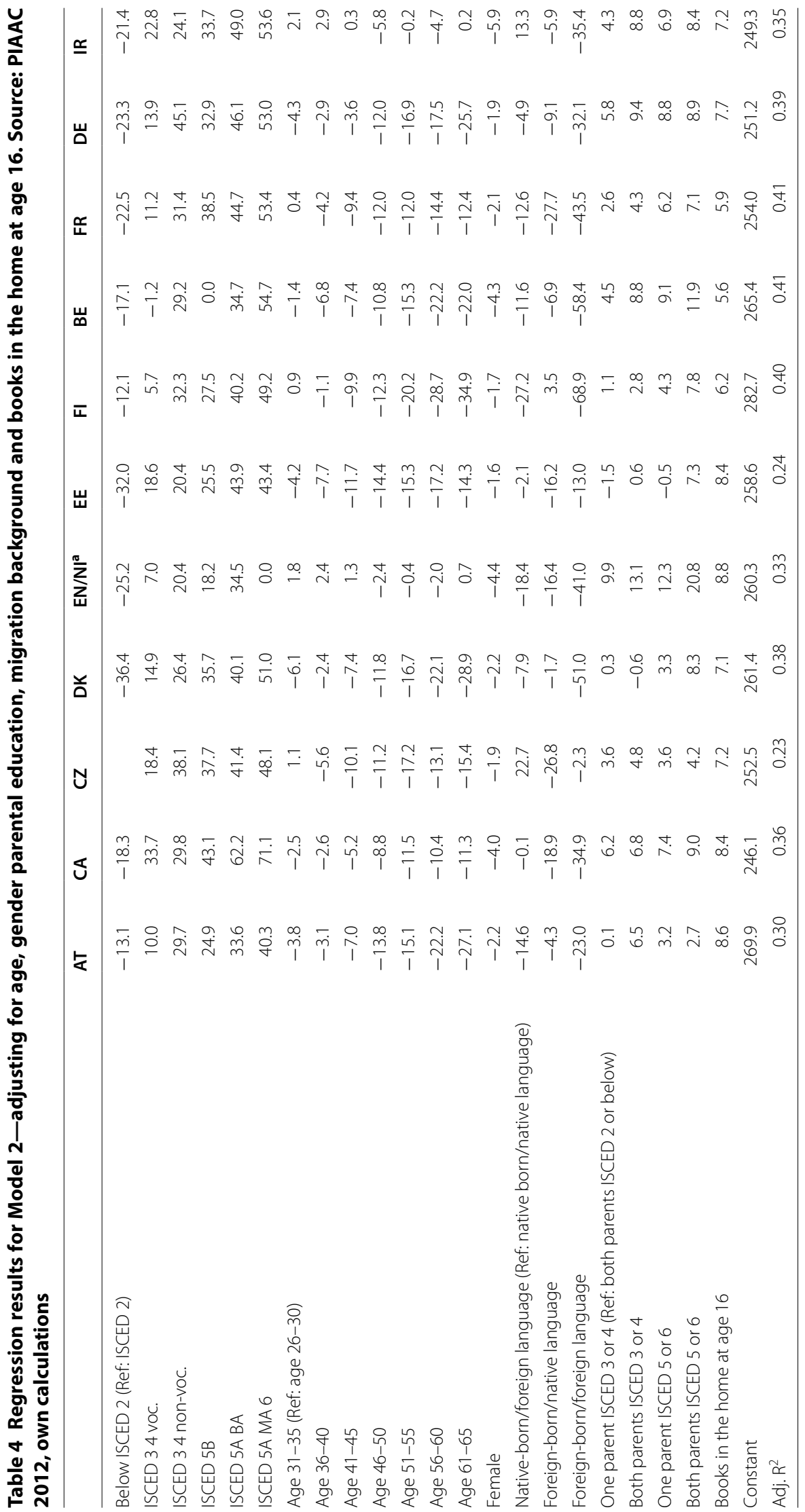




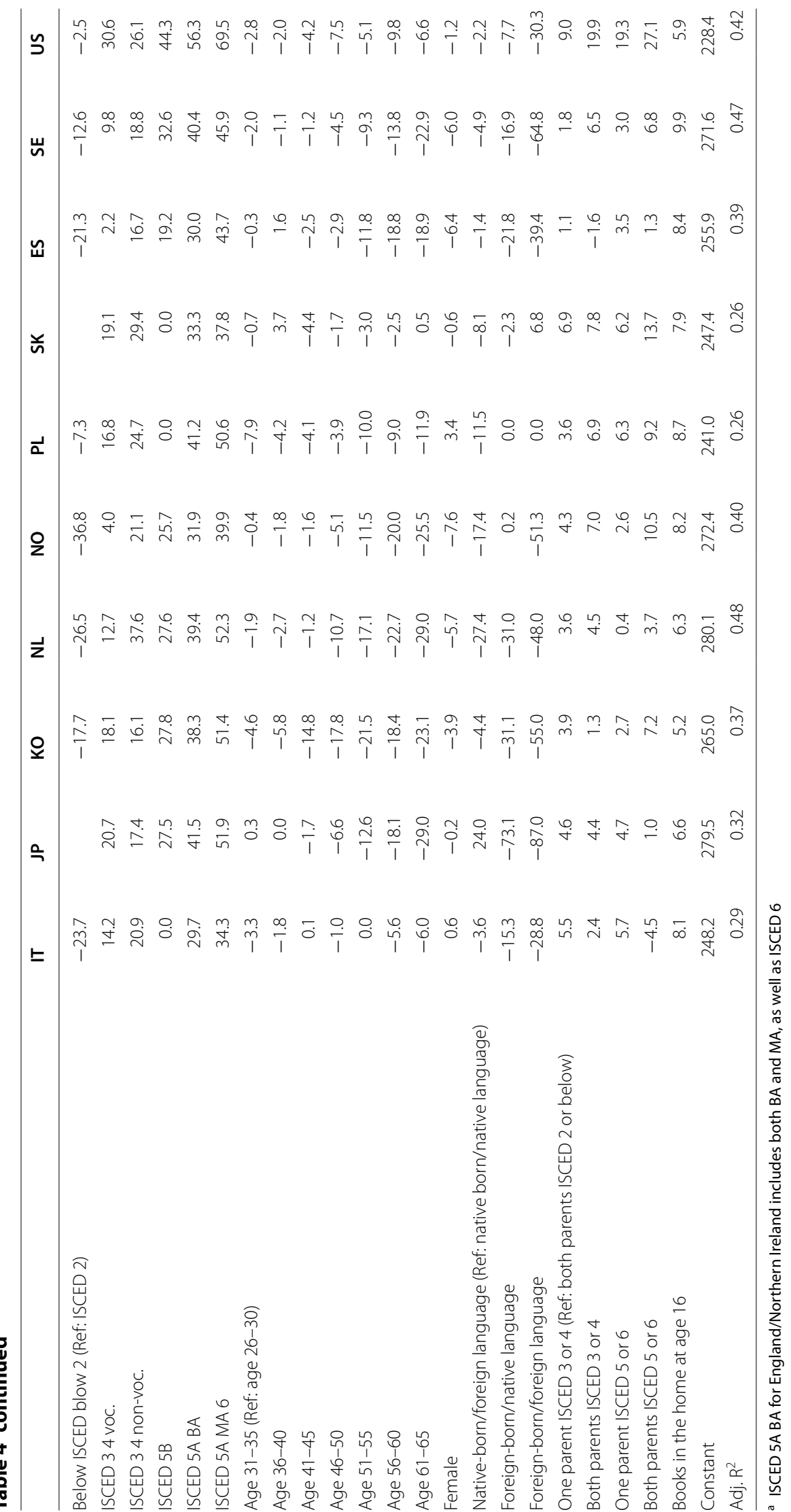




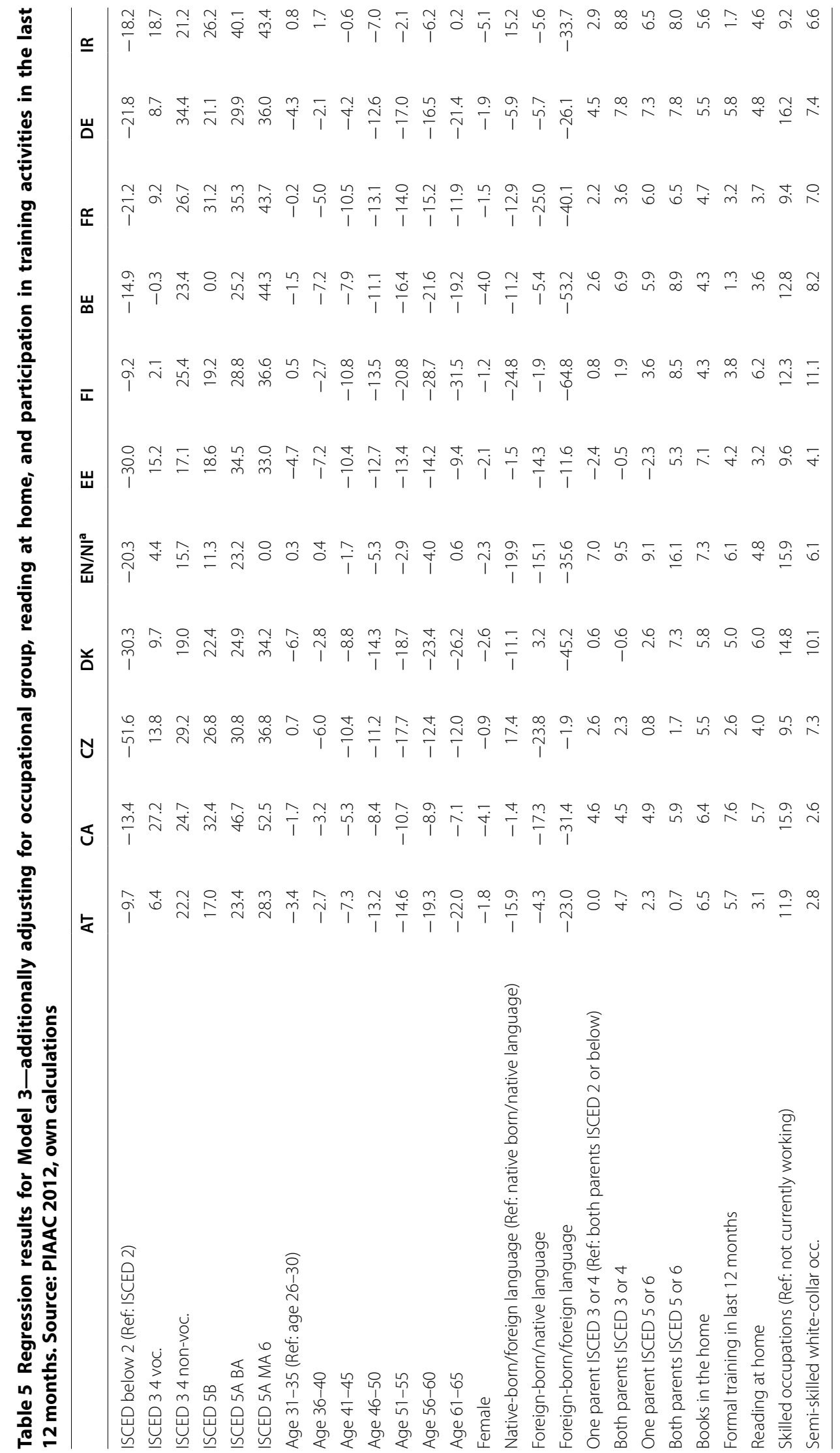




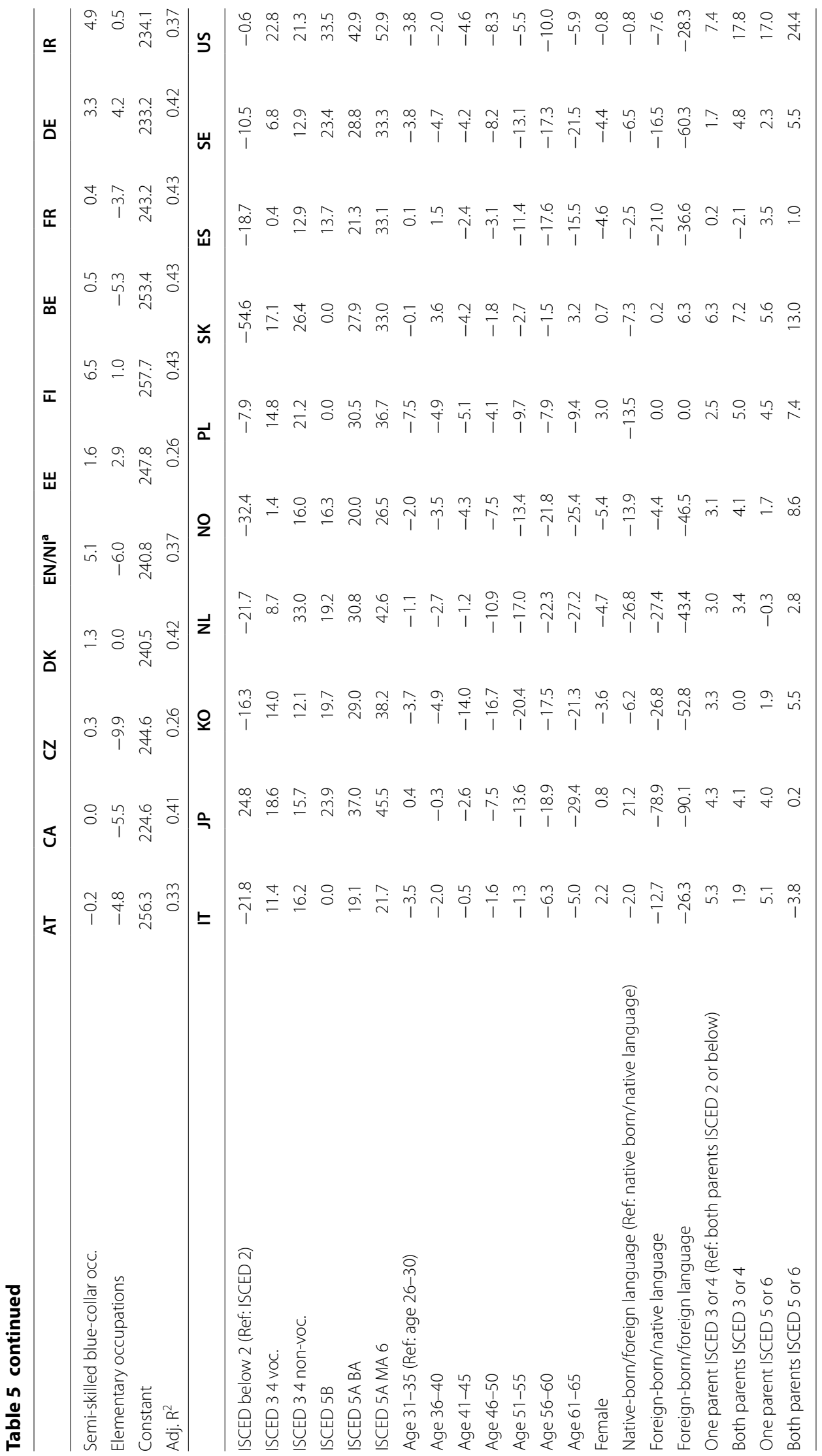




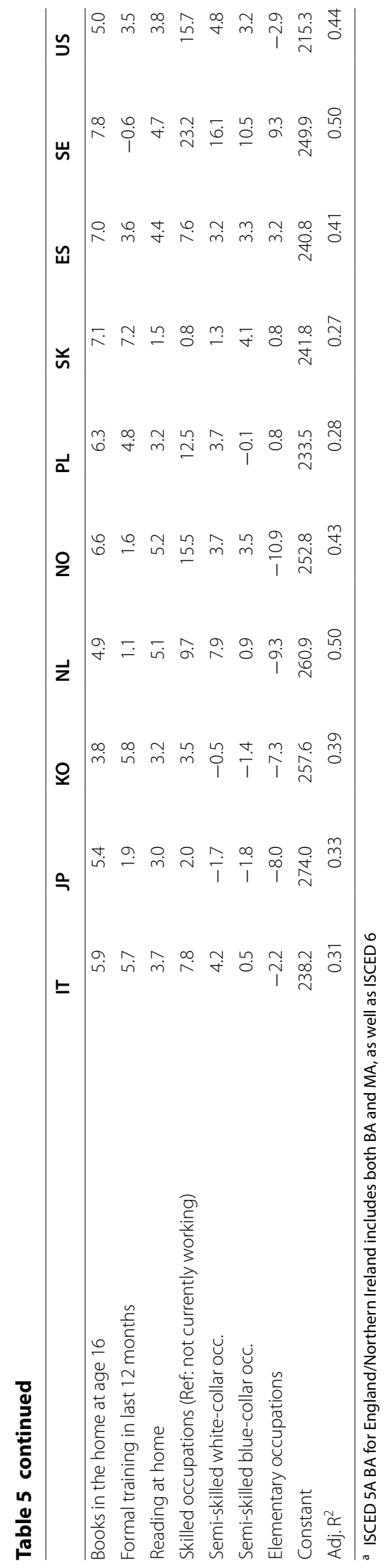


Received: 9 September 2016 Accepted: 24 January 2017

Published online: 07 February 2017

\section{References}

Allen, J., \& Van der Velden, R. (2001). Educational mismatches versus skill mismatches: Effects on wages, job satisfaction, and on-the-job search. Oxford Economic Papers, 53(3), 434-452. doi:10.1093/oep/53.3.434.

Anderson, R. C., Wilson, P. T., Fielding, L. G., Anderson, R. C., \& Fielding, L. G. (1988). Growth in reading and how children spend their time outside of school. Reading research Quarterly, 23(3), 285-303.

Arrow, K. (1973). Higher education as a filter. Journal of Public Economics, 2, 193-216.

Barrett, G., \& Riddell, W. C. (2016). Ageing and literacy skills: Evidence from IALS, ALL and PIAAC. IZA Discussion Papers, 10017. Institute for the Study of Labor (IZA), Bonn.

Baumert, J., Lüdtke, O., Trautwein, U., \& Brunner, M. (2009). Large-scale student assessment studies measure the results of processes of knowledge acquisition: Evidence in support of the distinction between intelligence and student achievement. Educational Research Review, 4(3), 165-176. doi:10.1016/j.edurev.2009.04.002.

Becker, G. S. (1964). Human capital: A theoretical and empirical analysis, with special reference to education. New York, NY: National Bureau of Economic Research.

Boudard, E. (2001). Literacy proficiency, earnings and recurrent training. A ten country comparative study. Institute of International Education, Stockholm.

Bourdieu, P. (1986). The forms of capital. In J. Richardson (Ed.), Handbook of theory and research for the sociology of education (pp. 241-258). New York: Greenwood.

Breen, R., \& Jonsson, J. O. (2005). Inequality of opportunity in comparative perspective: Recent research on educational attainment and social mobility. Annual Review of Sociology, 31(1), 223-243.

Breen, R., Luijkx, R., Müller, W., \& Pollak, R. (2010). Long-term trends in educational inequality in Europe: Class inequalities and gender differences. European Sociological Review, 26(1), 31-48.

Bynner, J., \& Parsons, S. (2009). Insights into basic skills from a UK longitudinal study. In S. Reder \& J. Bynner (Eds.), Tracking adult literacy and numeracy skills: Findings from longitudinal research (pp. 27-58). New York, NY: Taylor \& Francis.

Collins, R. (1971). Functional and conflict theories of educational stratification. American Sociological Review, 36(6), $1002-1019$.

Desjardins, R. (2003). Determinants of literacy proficiency: A lifelong-lifewide learning perspective. International Journal of Educational Research, 39(3), 205-245.

Desjardins, R. (2004). Learning for well being: studies using the International Adult Literacy Survey. No. 65 (R. Desjardins ed.) Stockholm: Stockholm University.

Desjardins, R., Rubenson, K., \& Milana, M. (2006). Unequal chances to participate in adult learning: International perspectives. Paris: UNESCO.

Desjardins, R., \&Warnke, A. (2012). Ageing and skills: A review and analysis of skill gain and skill loss over the lifespan and over time, OECD Education Working Papers, 72. Paris: OECD Publishing. doi:10.1787/5k9csvw87ckh-en.

Elley, W. B. (1992). How in the world do students read? IEA study of reading literacy. The Hague: International Association for the Evaluation of Educational Achievement.

Elwert, F., \& Winship, C. (2013). Endogenous selection bias: The problem of conditioning on a collider variable. Annual Review of Sociology, 40(1), 31-53. doi:10.1146/annurev-soc-071913-043455.

Erikson, R., \& Jonsson, J. O. (1996). Introduction. In R. Erikson \& J. O. Jonsson (Eds.), Explaining class inequality in education: The Swedish test case (pp. 1-63). Boulder (CO), Oxford: Westview.

Evans, M. D. R., Kelley, J., Sikora, J., \& Treiman, D. J. (2010). Family scholarly culture and educational success: Books and schooling in 27 nations. Research in Social Stratification and Mobility, 28(2), 171-197. doi:10.1016/j.rssm.2010.01.002.

Green, F., \& McIntosh, S. (2007). Is there a genuine under-utilization of skills amongst the over-qualified? Applied Economics, 39(4), 427-439. doi:10.1080/00036840500427700.

Heath, A. F., \& Brinbaum, Y. (Eds.). (2014). Unequal Attainments: Ethnic educational inequalities in ten Western countries. Oxford: Oxford University Press.

Heath, A. F., Rothon, C., \& Kilpi, E. (2008). The second generation in Western Europe: Education, unemployment, and occupational attainment. Annual Review of Sociology, 34(1), 211-235. doi:10.1146/annurev.soc.34.040507.134728.

Heisig, J. P., \& Solga, H. (2015). Secondary education systems and the general skills of less-and intermediate-educated adults a comparison of 18 countries. Sociology of Education, 88(3), 202-225.

International Labour Organisation. (2007). Resolution concerning updating the international standard classification of occupations. Retrieved from http://www.ilo.org/public/english/bureau/stat/isco/docs/resol08.pdf.

Kerckhoff, A. C., Raudenbush, S. W., \& Glennie, E. (2001). Education, cognitive skill, and labor force outcomes. Sociology of Education, 74(1), 1-24.

Kirsch, I. S., Jungeblut, A., Jenkins, L., \& Kolstad, A. (1993). Literacy in America: A first look at the results of the national adult literacy survey. Washington, D.C.: National Center for Education Statistics.

Maehler, D. B., Massing, N., Helmschrott, S., Rammstedt, B., Staudinger, U. M., \&Wolf, C. (2013). Grundlegende Kompetenzen in verschiedenen Bevölkerungsgruppen. In B. Rammstedt (Ed.), Grundlegende Kompetenzen Erwachsener im internationalen Vergleich: Ergebnisse von PIAAC 2012 (pp. 77-124). Münster: Waxmann.

Marks, G. N. (2005). Accounting for immigrant non-immigrant differences in reading and mathematics in twenty countries. Ethnic and Racial Studies, 28(5), 925-946. doi:10.1080/01419870500158943.

Marks, G. N. (2014). Education, social background and cognitive ability: The decline of the social. Abingdon, New York: Routledge.

Müller, W., \& Klein, M. (2008). Schein oder Sein: Bildungsdisparitäten in der Europäischen Statistik: Eine Illustration am Beispiel Deutschlands. Schmollers Jahrbuch, 128(4), 511-543.

OECD. (2011). How do girls compare to boys in reading skills PISA 2009 at a Glance. Paris: OECD. 
OECD. (2012). Settling in: OECD indicators of immigrant integration 2012. Paris: OECD.

OECD. (2013a). OECD Skills outlook 2013: First results from the survey of adult skills. Paris: OECD.

OECD. (2013b). The survey of adult skills—Reader's companion. Paris: OECD.

OECD. (2013c). Technical report of the survey of adult skills (PIAAC). Retrieved from Paris.

OECD. (2015). The ABC of gender equality in education: Aptitude, behaviour, confidence, PISA. Retrieved from.

OECD, \& Statistics Canada. (2000a). Literacy in the information age: Final report of the international adult literacy survey. Paris: OECD.

OECD, \& Statistics Canada. (2000b). Literacy in the information age: Final report of the international adult literacy survey (OECD Ed.). Paris: OECD.

OECD, \& Statistics Canada. (2005). Learning a living: First results of the adult literacy and life skills survey. Paris: OECD.

Park, H., \& Kyei, P. (2011). Literacy gaps by educational attainment: A cross-national analysis. Social Forces, 89(3), 879-904.

PIAAC Literacy Expert Group (2009). PIAAC Literacy: A Conceptual Framework. OECD Education Working Papers, 34. Paris: OECD Publishing. doi:10.1787/220348414075.

Rammstedt, B., Zabal, A., Martin, S., Perry, A., Helmschrott, S., Massing, N., et al. (2015). Programme for the international assessment of adult competencies (PIAAC), Germany — Reduced Version (2.1.0 ed.). Cologne: GESIS Data Archive.

Raudenbush, S. W., \& Kim, J.S. (2002). Statistical issues in analysis of international comparisons of educational achievement. In A. C. Porter \& A. Gamoran (Eds.), Methodological Advances in Cross-National Surveys of Educational Achievement (pp. 267-294). Washington DC: National Academy Press.

Reder, S. (1994). Practice-engagement theory: A sociocultural approach to literacy across languages and cultures. In B. M. Ferdman, R.-M. Weber, \& A. G. Ramirez (Eds.), literacy across languages and cultures (pp. 33-74). Albany, NY: State University of New York Press.

Reder, S. (1998). Literacy selection and literacy development: Structural equation models of the reciprocal effects of education and literacy. In M. C. Smith (Ed.) Literacy for the twenty-first century: Research policy, practices and the national adult literacy survey (pp. 139-157). Westport, CT: Praeger.

Reder, S. (2009). The development of literacy and numeracy in adult life. In S. Reder \& J. Bynner (Eds.), Tracking adult literacy and numeracy skills: Findings from longitudinal research (pp. 59-84). New York, NY: Taylor \& Francis.

Schneider, S. L. (2009). Confusing credentials: The cross-nationally comparable measurement of educational attainment: DPhil thesis. Oxford: University of Oxford.

Schneider, S. L. (2010). Nominal comparability is not enough: (In-)equivalence of construct validity of cross-national measures of educational attainment in the European Social Survey. Research in Social Stratification and Mobility, 28(3), 343-357. doi:10.1016/j.rssm.2010.03.001.

Schneider, S. L., Joye, D., \& Wolf, C. (2016). When translation is not enough: Background variables in comparative surveys. In C. Wolf, D. Joye, T. W. Smith, \& Y.-C. Fu (Eds.), The Sage Handbook of Survey Methodology (pp. 288-307). Los Angeles.

Shavit, Y., \& Blossfeld, H.-P. (1993). Persistent inequality: Changing educational attainment in thirteen countries. Boulder, CO: Westview Press.

Shavit, Y., \& Müller, W. (Eds.). (1998). From school to work: A comparative study of educational qualifications and occupational destinations. Oxford: Clarendon Press.

Smith, M. C. (1996). Differences in adults' reading practices and literacy proficiencies. Reading Research Quarterly, 31(2), 196-219. doi:10.1598/RRQ.31.2.5.

Spence, M. (1973). Job market signaling. The Quarterly Journal of Economics, 87(3), 355-374.

Steedman, H., \& McIntosh, S. (2001). Measuring low skills in Europe: How useful is the ISCED framework? Oxford Economic Papers, 53(3), 564-581. doi:10.1093/oep/53.3.564.

Steedman, H., \& Murray, A. (2001). Skill profiles of France, Germany, the Netherlands, Portugal, Sweden and the UK. European Journal for Vocational Training, 22(1), 3-14.

UNESCO Institute for Statistics. (2006). International Standard Classification of Education ISCED 1997. Retrieved from UNESCO Institute for Statistics (UNESCO-UIS): Retrieved from http://www.uis.unesco.org/Library/Documents/ isced97-en.pdf.

Van de Kerckhove, W., Mohadjer, L., \& Krenzke, T. (2013). Treatment of outcome-related non-response in an international literacy survey. Paper presented at the American Statistical Association Joint Statistical Meetings, Montreal, Canada.

Wolf, C., Schneider, S. L., Behr, D., \& Joye, D. (2016). Harmonizing survey questions between cultures and over time. In: C. Wolf, D. Joye, T. W. Smith, \& Y.-c. Fu (Eds.), The SAGE handbook of survey methodology (pp. 502-524). Los Angeles: Sage.

\section{Submit your manuscript to a SpringerOpen ${ }^{\circ}$ journal and benefit from:}

- Convenient online submission

- Rigorous peer review

- Immediate publication on acceptance

- Open access: articles freely available online

- High visibility within the field

- Retaining the copyright to your article

Submit your next manuscript at $\boldsymbol{\nabla}$ springeropen.com 